\title{
Di-neutron correlation in monopole two-neutron transfer modes in Sn isotope chain
}

\author{
Hirotaka Shimoyama, Masayuki Matsuo \\ Department of Physics, Faculty of Science and Graduate School of Science and Technology, \\ Niigata University, Niigata 950-2181, Japan
}

(Dated: October 23, 2018)

\begin{abstract}
We study microscopic structures of monopole pair vibrational modes and associated two-neutron transfer amplitudes in neutron-rich Sn isotopes by means of the linear response formalism of the quasiparticle random phase approximation(QRPA). For this purpose we introduce a method to decompose the transfer amplitudes with respect to two-quasiparticle components of the QRPA eigen mode. It is found that pair-addition vibrational modes in neutron-rich ${ }^{132-140} \mathrm{Sn}$ and the pair rotational modes in ${ }^{142-150} \mathrm{Sn}$ are commonly characterized by coherent contributions of quasaiparticle states having high orbital angular momenta $l \gtrsim 5$, which suggests transfer of a spatially correlated neutron pair. The calculation also predicts a high-lying pair vibration, the giant pair vibration, emerging near the one-neutron separation energy in ${ }^{110-130} \mathrm{Sn}$, and we find that they have the same di-neutron characters as that of the low-lying pair vibration in ${ }^{132-140} \mathrm{Sn}$.
\end{abstract}

PACS numbers: 21.10.Pc,21.10.Re,21.60.Jz,24.30.Cz,25.40.Hs 


\section{INTRODUCTION}

Nuclei close to the drip-lines often exhibit exotic features which are not present in stable nuclei. Representative examples are the neutron halo and skin [1, 2], which are associated with the weak binding of the last neutrons. Another example is the neutron pair correlation, which may be enhanced in the surface region with the skin or halo[3 [5]. The neutron pairing in the low density surface region may be related to the predicted strong density dependence of the pairing in neutron matter $6-10]$.

Because of the low-density enhancement, the pair correlation in neutron-rich nuclei may lead to the di-neutron correlation, i.e., a strong spatial correlation among the two neutrons of the neutron Cooper pair. The di-neutron correlation has been discussed in the two-neutron halo nuclei, e.g. ${ }^{11} \mathrm{Li}[3,11$ 18], and recently its possibility is extended for heavier-mass pair correlated nuclei[19 23]. The spatial correlation has been discussed also for the closedshell plus two-neutron (two-neutron hole) configuration, e.g. ${ }^{210} \mathrm{~Pb}[24-26]$. It is argued also that the di-neutron correlation affects the nature of excitation modes, a typical example of which is the soft dipole excitation in the two-neutron halo nucleus ${ }^{11} \mathrm{Li}[3$, 15, 18, 27]. It is also predicted that the soft octupole excitation in medium heavy neutron-rich nuclei, e.g. ${ }^{84} \mathrm{Ni}[28]$, and excited states of clustering nuclei, e.g. ${ }^{10} \mathrm{Be}[29]$, exhibit the di-neutron feature.

The most direct influence of the pair correlation is expected to be seen in transfers of two nucleons. The pair correlation induces collective excitation modes, known as the pair vibration and the pair rotation, which accompany enhanced two-nucleon transfer reaction cross sections [30 34]. Recently, the two-neutron transfer experiments have been conducted for some neutron-rich nuclei[35-40]. Motivated by the above mentioned interests and prospects of future experiments, theoretical studies of the two-neutron transfers in neutron rich nuclei also have been performed[41 48].

We have been investigating the two-neutron transfer modes in neutron-rich Sn isotopes in order to explore possible new features of the pair vibration and the pair rotation[44, 45]. We adopt the nuclear energy density functional model, in particular, the Skyrme-HartreeFock-Bogoliubov model to describe the pair correlated ground state and the associated pair rotational two-neutron transfer mode, and we apply the quasiparticle random phase approximation (QRPA) to study the pair vibrations and the associated two-neutron transfer amplitudes. In a preceding work[45], where we described monopole low-lying pair vibration 
in the whole isotope chain $100 \leq A \leq 150$, we found a characteristic pair vibration in $132 \leq A \leq 140$, which has a large pair-addition strength, comparable to that of the groundstate pair-rotational mode, and much larger than the independent-particle transitions. An interesting feature is that the transition density of this pair vibration mode has a long tail which reaches far outside the nuclear surface $r \sim 15 \mathrm{fm}$.

One of the purposes of the present paper is to clarify the nature of the characteristic pair vibration predicted in our previous study[45] for neutron-rich $\mathrm{Sn} A=132-140$ isotopes. We shall analyze in detail the microscopic structure of this mode by looking into the single-particle compositions. We decompose the transition density and the QRPA phonon amplitude with respect to two-quasiparticle configurations forming the QRPA phonon. As shown later, we will find that a large number of both weakly bound and unbound quasineutron states contribute coherently to build up the characteristic correlation in this mode. We will find further that this correlation is consistent with what we expect for a spatially correlated neutron-pair, the di-neutron, which is transfered in the pair vibration.

In the previous paper [45], we found also that the pair-transfer strength associated with the pair rotation (the transition between the ground states of the neighboring even nuclei) significantly increases as the neutron number further increases, i.e., in the isotopes beyond $A=140$. In the present paper, we shall also analyze microscopic structure of this enhanced ground state transfer. We will show that it is indeed related to the characteristic pair vibration in ${ }^{132-140} \mathrm{Sn}$. In the present study, we also aim at widening the scope of our study of the pairing collectivity. Namely, we perform our numerical calculation in a very wide interval $A=100 \sim 150$ of the Sn isotopic chain, including the stable isotopes, and we explore high excitation energy region $\sim 10-20 \mathrm{MeV}$, where we can expect the giant pair vibration. We will discuss how the giant pair vibration, which we find in the isotopes $A=110-130$, is related to the low-lying pair vibration and the pair rotation in the very neutron-rich nuclei $A=132-140$ and $A>140$. 


\section{TWO-QUASIPARTICLE COMPONENTS OF THE QRPA MODE}

\section{A. Linear response formulation of the QRPA}

In the present study, we adopt the Skyrme-Hartree-Fock-Bogoliubov model to describe the pair correlated ground states, and apply a linear response formulation of the quasiparticle

random phase approximation (QRPA) to describe the excitation modes [28, 44, 45, 49]. The framework is essentially the same as what we have adopted in the preceding study of the two-neutron transfer in neutron-rich Sn isotopes [44, 45]. Here we recapitulate only the basic framework with emphasis on new aspects of the formulation in the present work.

We describe the nuclear response at the frequency $\omega$ by solving the linear response equation

$$
\delta \rho_{\alpha L}(r, \omega)=\int_{0} d r^{\prime} \sum_{\beta} R_{0, L}^{\alpha \beta}\left(r, r^{\prime}, \omega\right)\left(\sum_{\gamma} \frac{\partial v_{\beta}}{\partial \rho_{\gamma}}\left(r^{\prime}\right) \frac{1}{r^{\prime 2}} \delta \rho_{\gamma L}\left(r^{\prime}, \omega\right)+v_{\beta L}^{e x t}\left(r^{\prime}\right)\right)
$$

for three kinds of densities $\delta \rho_{\alpha L}(r, \omega)=\delta \rho_{L}(r, \omega)$ and $\delta \tilde{\rho}_{ \pm L}(r, \omega)$, which are fluctuating parts of the normal density and the two kinds of pair densities;

$$
\begin{aligned}
\rho(\boldsymbol{r}) & =\langle 0|\hat{\rho}(\boldsymbol{r})| 0\rangle \\
& =\left\langle 0\left|\sum_{\sigma} \psi^{\dagger}(\boldsymbol{r} \sigma) \psi(\boldsymbol{r} \sigma)\right| 0\right\rangle, \\
\tilde{\rho}_{ \pm}(\boldsymbol{r}) & =\left\langle 0\left|\hat{\tilde{\rho}}_{ \pm}(\boldsymbol{r})\right| 0\right\rangle \\
& =\left\langle 0\left|\psi^{\dagger}(\boldsymbol{r} \downarrow) \psi^{\dagger}(\boldsymbol{r} \uparrow) \pm \psi(\boldsymbol{r} \uparrow) \psi(\boldsymbol{r} \downarrow)\right| 0\right\rangle .
\end{aligned}
$$

The unperturbed response function $R_{0, q L}^{\alpha \beta}\left(r, r^{\prime}, \omega\right)$ is expressed as

$$
\begin{aligned}
R_{0, L}^{\alpha \beta}\left(r, r^{\prime}, \omega\right)= & \frac{1}{2} \sum_{i \geq i^{\prime}} \frac{\left|\left\langle l^{\prime} j^{\prime}|| Y_{L}|| l j\right\rangle\right|^{2}}{2 L+1} \\
& \times\left\{\frac{\left\langle 0\left|\rho_{\alpha}(r)\right| i i^{\prime}\right\rangle\left\langle i i^{\prime}\left|\rho_{\beta}\left(r^{\prime}\right)\right| 0\right\rangle}{\hbar \omega+i \epsilon-E_{i}-E_{i^{\prime}}}-\frac{\left\langle 0\left|\rho_{\beta}\left(r^{\prime}\right)\right| i i^{\prime}\right\rangle\left\langle i i^{\prime}\left|\rho_{\alpha}(r)\right| 0\right\rangle}{\hbar \omega+i \epsilon+E_{i}+E_{i^{\prime}}}\right\},
\end{aligned}
$$

with

$$
\begin{aligned}
\left\langle i i^{\prime}\left|\rho_{\alpha}(r)\right| 0\right\rangle & \equiv \phi_{i}^{\mathrm{T}}(r) \mathcal{A} \bar{\phi}_{i^{\prime}}(r), \\
\left\langle 0\left|\rho_{\alpha}(r)\right| i i^{\prime}\right\rangle & \equiv \bar{\phi}_{i^{\prime}}^{\mathrm{T}}(r) \mathcal{A} \phi_{i}(r),
\end{aligned}
$$

if we adopt the spectral representation. Here $\mathcal{A}$ is $2 \times 2$ matrices which correspond to the three kinds of densities $\rho_{\alpha}(\boldsymbol{r})=\rho(\boldsymbol{r})$ and $\tilde{\rho}_{ \pm}(\boldsymbol{r})$. Index $i$ is a quantum number $[n l j]_{q}(q=\mathrm{n}, \mathrm{p})$ of the 
one-quasiparticle state whose energy is $E_{i}$ and wave function is $\phi_{i}(\boldsymbol{r} \sigma)=Y_{l j m}(\hat{\boldsymbol{r}} \sigma) \phi_{i}(r) / r$ where $\phi_{i}(\hat{\boldsymbol{r}} \sigma)$ and its radial part $\phi_{i}(r)$ have two components, $\phi_{i}(r)=\left[\varphi_{1, i}(r), \varphi_{2, i}(r)\right]^{T}$.

In the preceding paper[45], we adopted the continuum version of the linear response QRPA, i.e. the continuum QRPA[49], in which the unperturbed response function is constructed in terms of the HFB Green's function in place of the spectral representation. The continuum QRPA is appropriate to describe the response at energies above the particleemission threshold. However in this present work, we use the unperturbed response function of Eq. (6) together with the discretized quasiparticle eigenstates of the HFB equation. As shown below, this enables us to express the microscopic structure of the QRPA phonon and the transition densities in terms of two-quasiparticle components.

\section{B. Two-quasiparticle components of the QRPA phonon}

It is customary to expresses the QRPA phonon operator as a superposition of twoquasiparticle creation and annihilation operators:

$$
O_{L M}^{\nu \dagger}=\sum_{i \geq i^{\prime}} \frac{1}{\sqrt{1+\delta_{i i^{\prime}}}}\left\{X_{i i^{\prime}}^{\nu}\left[a_{i}^{\dagger} a_{i^{\prime}}^{\dagger}\right]_{L M}-Y_{i i^{\prime}}^{\nu}\left[a_{i^{\prime}} a_{i}\right]_{L M}\right\},
$$

with the two-quasiparticle operators defined by

$$
\begin{aligned}
{\left[a_{i}^{\dagger} a_{i^{\prime}}^{\dagger}\right]_{L M} } & \equiv \sum_{m m^{\prime}}\left\langle j m j^{\prime} m^{\prime} \mid L M\right\rangle a_{n l j m}^{\dagger} a_{n^{\prime} l^{\prime} j^{\prime} m^{\prime}}^{\dagger} \\
{\left[a_{i} a_{i^{\prime}}\right]_{L M} } & \equiv \sum_{m m^{\prime}}\left\langle j m j^{\prime} m^{\prime} \mid L M\right\rangle \tilde{a}_{n l j m} \tilde{a}_{n^{\prime} l^{\prime} j^{\prime} m^{\prime}}
\end{aligned}
$$

where $X_{i i^{\prime}}^{\nu}$ and $Y_{i i^{\prime}}^{\nu}$ are forward and backward amplitudes of the two-quasiparticle component $i i^{\prime}$. Index $\nu$ stands for the QRPA normal mode whose excitation energy is denoted as $\hbar \omega_{\nu}$. For the time-reversal convention, we employ $T a_{n l j m} T^{\dagger} \equiv \tilde{a}_{n l j m}=(-)^{l-j+m} a_{n l j-m}$.

The $X$ - and $Y$-amplitudes are often obtained by diagonalization of the QRPA matrix [50]. Below, we shall show that one can also calculate the $X$-and $Y$-amplitudes on the basis of the linear response formalism.

Let us consider transition densities

$$
\rho_{\alpha L M}^{(\operatorname{tr}) \nu}(\boldsymbol{r})=\left\langle 0\left|\hat{\rho}_{\alpha}(\boldsymbol{r})\right| \nu\right\rangle,
$$

for the three kinds of densities $\hat{\rho}_{\alpha}(\boldsymbol{r})=\hat{\rho}(\boldsymbol{r}), \hat{\tilde{\rho}}_{ \pm}(\boldsymbol{r})$. This quantity can be expressed in the following two ways. First, using the phonon creation operator $O_{L M}^{\nu \dagger}$ that describes the 
excited state $|\nu\rangle$ as $|\nu\rangle=O_{L M}^{\nu \dagger}|0\rangle$, Eq. (12) is written as

$$
\begin{aligned}
\rho_{\alpha L M}^{(\operatorname{tr}) \nu}(\boldsymbol{r})= & \left\langle 0\left|\left[\hat{\rho}_{\alpha}(\boldsymbol{r}), O_{L M}^{\nu \dagger}\right]\right| 0\right\rangle \\
= & \sum_{i \geq i^{\prime}} \frac{1}{\sqrt{1+\delta_{i i^{\prime}}}}\left\{X_{i i^{\prime}}^{\nu}\left\langle 0\left|\left[\hat{\rho}_{\alpha}(\boldsymbol{r}),\left[a_{i}^{\dagger} a_{i^{\prime}}^{\dagger}\right]_{L M}\right]\right| 0\right\rangle-Y_{i i^{\prime}}^{\nu}\left\langle 0\left|\left[\hat{\rho}_{\alpha}(\boldsymbol{r}),\left[a_{i^{\prime}} a_{i}\right]_{L M}\right]\right| 0\right\rangle\right\}(16) \\
= & Y_{L M}(\hat{\boldsymbol{r}}) \frac{1}{r^{2}} \sum_{i \geq i^{\prime}} \frac{1}{\sqrt{1+\delta_{i i^{\prime}}}}\left\{(-)^{l^{\prime}} X_{i i^{\prime}}^{\nu} \frac{\left\langle l^{\prime} j^{\prime}|| Y_{L}|| l j\right\rangle^{*}}{\sqrt{2 L+1}}\left\langle 0\left|\rho_{\alpha}(r)\right| i i^{\prime}\right\rangle\right. \\
& \left.+(-)^{l} Y_{i i^{\prime}}^{\nu} \frac{\left\langle l^{\prime} j^{\prime}|| Y_{L}|| l j\right\rangle^{*}}{\sqrt{2 L+1}}\left\langle i i^{\prime}\left|\rho_{\alpha}(r)\right| 0\right\rangle\right\} .
\end{aligned}
$$

The other way is to use the density response $\delta \rho_{\alpha L}(r, \omega)$ of the linear response formalism. In the linear response formalism, the transition density can be calculated as

$$
\rho_{\alpha L M}^{(\operatorname{tr}) \nu}(\boldsymbol{r})=Y_{L M}(\hat{\boldsymbol{r}})\left[-\frac{C}{\pi r^{2}} \operatorname{Im} \delta \rho_{\alpha L}\left(r, \omega_{\nu}\right)\right]
$$

where $\omega_{\nu}$ is the frequency of the QRPA normal mode. We then rewrite this by using the linear response equation (Eq. (1)) and the unperturbed response function (Eq. ([6) ), and we obtain an expression for the transition density

$$
\begin{aligned}
\rho_{\alpha L M}^{(\operatorname{tr}) \nu}(\boldsymbol{r})=Y_{L M}(\hat{\boldsymbol{r}}) \frac{1}{r^{2}} & \times \sum_{i \geq i^{\prime}}\left\{\left[-\frac{C}{2 \pi} \frac{\left\langle l^{\prime} j^{\prime}|| Y_{L}|| l j\right\rangle^{2}}{2 L+1} \frac{1}{\hbar \omega_{\nu}-E_{i}-E_{i^{\prime}}}\right.\right. \\
& \left.\times \int_{0} d r^{\prime} \sum_{\beta}\left\langle i i^{\prime}\left|\rho_{\beta}\left(r^{\prime}\right)\right| 0\right\rangle\left\{\sum_{\gamma} \kappa_{\beta \gamma}\left(r^{\prime}\right) \operatorname{Im} \delta \rho_{\gamma L}\left(r^{\prime}, \omega_{\nu}\right)\right\}\right]\left\langle 0\left|\rho_{\alpha}(r)\right| i i^{\prime}\right\rangle \\
+ & {\left[\frac{C}{2 \pi} \frac{\left\langle l^{\prime} j^{\prime}|| Y_{L}|| l j\right\rangle^{2}}{2 L+1} \frac{1}{\hbar \omega_{\nu}+E_{i}+E_{i^{\prime}}}\right.} \\
& \left.\left.\times \int_{0} d r^{\prime} \sum_{\beta}\left\langle 0\left|\rho_{\beta}\left(r^{\prime}\right)\right| i i^{\prime}\right\rangle\left\{\sum_{\gamma} \kappa_{\beta \gamma}\left(r^{\prime}\right) \operatorname{Im} \delta \rho_{\gamma L}\left(r^{\prime}, \omega_{\nu}\right)\right\}\right]\left\langle i i^{\prime}\left|\rho_{\alpha}(r)\right| 0\right\rangle\right\} .
\end{aligned}
$$

Note that since the density response $\delta \rho_{\alpha L}(r, \omega)$ exhibits a pole behavior $\propto 1 /\left(\hbar \omega+i \epsilon-\hbar \omega_{\nu}\right)$ at frequencies near the QRPA eigen mode $\hbar \omega_{\nu}$, the external field $v_{\beta L}^{e x t}(r)$ in Eq. (1) is irrelevant here, provided that we take a sufficiently small value of $\epsilon$. The coefficient $C$ is a normalization constant.

Comparing the above two definitions of the transition density, Eq. (15) and Eq. (17), we obtain the expression for the forward and backward amplitudes $X_{i i^{\prime}}^{\nu}$ and $Y_{i i^{\prime}}^{\nu}$,

$$
X_{i i^{\prime}}^{\nu}=(-)^{l^{\prime}+1} \frac{C}{2 \pi} \frac{\left\langle l^{\prime} j^{\prime}|| Y_{L}|| l j\right\rangle}{\sqrt{2 L+1}} \frac{1}{\hbar \omega_{\nu}-E_{i}-E_{i^{\prime}}}
$$




$$
\begin{aligned}
& \times \int_{0} d r^{\prime} \sum_{\beta}\left\langle i i^{\prime}\left|\rho_{\beta}\left(r^{\prime}\right)\right| 0\right\rangle\left\{\sum_{\gamma} \frac{\partial v_{\beta}}{\partial \rho_{\gamma}}\left(r^{\prime}\right) \frac{1}{r^{\prime 2}} \operatorname{Im} \delta \rho_{\gamma L}\left(r^{\prime}, \omega_{\nu}\right)\right\}, \\
& Y_{i i^{\prime}}^{\nu}=(-)^{l} \frac{C}{2 \pi} \frac{\left\langle l^{\prime} j^{\prime}|| Y_{L}|| l j\right\rangle}{\sqrt{2 L+1}} \frac{1}{\hbar \omega_{\nu}+E_{i}+E_{i^{\prime}}} \\
& \times \int_{0} d r^{\prime} \sum_{\beta}\left\langle 0\left|\rho_{\beta}\left(r^{\prime}\right)\right| i i^{\prime}\right\rangle\left\{\sum_{\gamma} \frac{\partial v_{\beta}}{\partial \rho_{\gamma}}\left(r^{\prime}\right) \frac{1}{r^{\prime 2}} \operatorname{Im} \delta \rho_{\gamma L}\left(r^{\prime}, \omega_{\nu}\right)\right\} .
\end{aligned}
$$

The normalization constant $C$ is determined to fulfill the normalization condition $\left\langle 0\left|\left[O_{L M}^{\nu}, O_{L M}^{\nu \dagger}\right]\right| 0\right\rangle=\sum_{i \geq i^{\prime}}\left|X_{i i^{\prime}}^{\nu}\right|^{2}-\left|Y_{i i^{\prime}}^{\nu}\right|^{2}=1$.

\section{Decomposition of the transition densities}

Once the $X$ - and $Y$-amplitudes are obtained, it is straightforward to decompose the transition density with respect to the two-quasiparticle components of the QRPA phonon:

$$
\begin{aligned}
\rho_{\alpha L M}^{(\operatorname{tr}) \nu}(\boldsymbol{r}) & =\sum_{i \geq i^{\prime}} \rho_{\alpha L M, i i^{\prime}}^{(\operatorname{tr}) \nu}(\boldsymbol{r}) \\
\rho_{\alpha L M, i i^{\prime}}^{(\operatorname{tr}) \nu}(\boldsymbol{r}) & =\frac{1}{\sqrt{1+\delta_{i i^{\prime}}}}\left\{X_{i i^{\prime}}^{\nu}\left\langle 0\left|\left[\hat{\rho}_{\alpha}(\boldsymbol{r}),\left[a_{i}^{\dagger} a_{i^{\prime}}^{\dagger}\right]_{L M}\right]\right| 0\right\rangle-Y_{i i^{\prime}}^{\nu}\left\langle 0\left|\left[\hat{\rho}_{\alpha}(\boldsymbol{r}),\left[a_{i^{\prime}} a_{i}\right]_{L M}\right]\right| 0\right\rangle\right\} .
\end{aligned}
$$

Here $\rho_{\alpha L M, i i^{\prime}}^{(\operatorname{tr}) \nu}(\boldsymbol{r})$ is a partial contribution from a two-quasiparticle configuration $i i^{\prime}$. Its radial part is expressed as

$$
\begin{aligned}
\rho_{\alpha L M, i i^{\prime}}^{(\operatorname{tr}) \nu}(\boldsymbol{r}) & =Y_{L M}(\hat{\boldsymbol{r}}) \rho_{\alpha L, i i^{\prime}}^{(\operatorname{tr}) \nu}(r), \\
\rho_{\alpha L, i i^{\prime}}^{(\operatorname{tr}) \nu}(r) & =\frac{\left\langle l^{\prime} j^{\prime}|| Y_{L}|| l j\right\rangle^{*}}{\sqrt{2 L+1}} \frac{1}{r^{2}}\left\{(-)^{l^{\prime}} X_{i i^{\prime}}^{\nu}\left\langle 0\left|\rho_{\alpha}(r)\right| i i^{\prime}\right\rangle+(-)^{l} Y_{i i^{\prime}}^{\nu}\left\langle i i^{\prime}\left|\rho_{\alpha}(r)\right| 0\right\rangle\right\} .
\end{aligned}
$$

In the present paper, we describe the pair transfer modes in terms of operators

$$
\begin{aligned}
& P^{\dagger}(\boldsymbol{r})=\psi^{\dagger}(\boldsymbol{r} \downarrow) \psi^{\dagger}(\boldsymbol{r} \uparrow)=\frac{1}{2}\left(\hat{\tilde{\rho}}_{+}(\boldsymbol{r})-\hat{\tilde{\rho}}_{-}(\boldsymbol{r})\right), \\
& P(\boldsymbol{r})=\psi(\boldsymbol{r} \uparrow) \psi(\boldsymbol{r} \downarrow)=\frac{1}{2}\left(\hat{\tilde{\rho}}_{+}(\boldsymbol{r})+\hat{\tilde{\rho}}_{-}(\boldsymbol{r})\right),
\end{aligned}
$$

which adds and removes a spin-singlet $(S=0)$ neutron pair, respectively. We then describe transition densities for these pair-addition and pair-removal operators, defined by

$$
\begin{aligned}
& P_{\nu L M}^{(\mathrm{ad})}(\boldsymbol{r}) \equiv\langle 0|\psi(\boldsymbol{r} \downarrow) \psi(\boldsymbol{r} \uparrow)| \nu\rangle=P_{\nu L}^{(\mathrm{ad})}(r) Y_{L M}(\hat{\boldsymbol{r}}), \\
& P_{\nu L M}^{(\mathrm{rm})}(\boldsymbol{r}) \equiv\left\langle 0\left|\psi^{\dagger}(\boldsymbol{r} \downarrow) \psi^{\dagger}(\boldsymbol{r} \uparrow)\right| \nu\right\rangle=P_{\nu L}^{(\mathrm{rm})}(r) Y_{L M}(\hat{\boldsymbol{r}}),
\end{aligned}
$$

which are decomposed as

$$
P_{\nu L}^{(\mathrm{ad} / \mathrm{rm})}(r)=\sum_{i \geq i^{\prime}} P_{\nu L, i i^{\prime}}^{(\mathrm{ad} / \mathrm{rm})}(r)
$$


and

$$
\begin{aligned}
P_{\nu L, i i^{\prime}}^{(\mathrm{ad} / \mathrm{rm})}(r)=\frac{1}{\sqrt{1+\delta_{i i^{\prime}}}} \frac{\left\langle l^{\prime} j^{\prime}|| Y_{L}|| l j\right\rangle^{*}}{2 r^{2}}\left[(-)^{l^{\prime}} X_{i i^{\prime}}^{\nu}\left\{\left\langle 0\left|\tilde{\rho_{+}}(r)\right| i i^{\prime}\right\rangle \pm\left\langle 0\left|\tilde{\rho_{-}}(r)\right| i i^{\prime}\right\rangle\right\}\right. \\
\left.+(-)^{l} Y_{i i^{\prime}}^{\nu}\left\{\left\langle i i^{\prime}\left|\tilde{\rho_{+}}(r)\right| 0\right\rangle \pm\left\langle i i^{\prime}\left|\tilde{\rho_{-}}(r)\right| 0\right\rangle\right\}\right] .
\end{aligned}
$$

We can also consider the transition density of the pair-rotational ground-state transfer [45]. The transition density is also expanded using the quasiparticle states:

$$
\begin{aligned}
P_{\mathrm{gs}}^{(\mathrm{ad} / \mathrm{rm})}(\boldsymbol{r}) & =\left\langle 0\left|\psi^{\dagger}(\boldsymbol{r} \sigma) \psi^{\dagger}(\boldsymbol{r} \tilde{\sigma})\right| 0\right\rangle \\
& =-\left\langle 0\left|\sum_{i i^{\prime}} \sum_{m m^{\prime}} \varphi_{2, i}(\boldsymbol{r} \tilde{\sigma}) \varphi_{1, i^{\prime}}^{*}(\boldsymbol{r} \tilde{\sigma}) a_{i} a_{i^{\prime}}^{\dagger}\right| 0\right\rangle \\
& =-\frac{1}{4 \sqrt{\pi}} \frac{1}{r^{2}} \sum_{i} \varphi_{2, i}(r) \varphi_{1, i}^{*}(r) Y_{00}(\hat{\boldsymbol{r}})
\end{aligned}
$$

Then, the radial transition density of the ground state transfer and its decomposition are

$$
\begin{aligned}
& P_{\mathrm{gs}}^{(\mathrm{ad} / \mathrm{rm})}(r)=\sum_{i} P_{\mathrm{gs}, i i}^{(\mathrm{ad} / \mathrm{rm})}(r), \\
& P_{\mathrm{gs}, i i}^{(\mathrm{ad} / \mathrm{rm})}(r)=-\frac{1}{4 \sqrt{\pi}} \frac{1}{r^{2}}(2 j+1) \varphi_{2, i}(r) \varphi_{1, i}(r) .
\end{aligned}
$$

\section{Details of calculation}

We assume the spherical ground states for the proton closed-shell nuclei $\operatorname{Sn}(Z=50)$. The Skyrme interaction parameter SLy4 [51] is chosen for the particle-hole channel of the HFB equations. The Landau-Migdal approximation is employed for the RPA residual interaction in the particle-hole channel. For the pairing interaction we adopt density-dependent delta interaction (DDDI) [4, 12, 52, 53]. The DDDI is given by $v_{q}^{\text {pair }}\left(\boldsymbol{r}, \boldsymbol{r}^{\prime}\right)=\frac{1}{2} V_{q}(\boldsymbol{r}) \delta\left(\boldsymbol{r}-\boldsymbol{r}^{\prime}\right)$, where $V_{q}(\boldsymbol{r})$ is the pairing interaction strength and is a function of the neutron and proton densities. We adopt the following form:

$$
V_{q}(\boldsymbol{r})=v_{0}\left[1-\eta\left(\frac{\rho_{q}(\boldsymbol{r})}{\rho_{0}}\right)^{\alpha}\right] .
$$

The value $v_{0}=-458.4 \mathrm{MeV} \mathrm{fm}^{3}$ is chosen to reproduce the scattering length $a=-18.5 \mathrm{fm}$ of the bare neutron-neutron interaction in the ${ }^{1} S$ channel. The parameter $\eta=0.71$ is adjusted to reproduce the experimental pairing gap in ${ }^{120} \mathrm{Sn}$. The other parameters are $\rho_{0}=0.08$ $\mathrm{fm}^{-3}$ and $\alpha=0.59[8$, 54].

We solve the HFB equation in the coordinate space representation using the polar coordinate system. The radial coordinate space is truncated at $r_{\max }=20 \mathrm{fm}$. We truncate the 
quasiparticle states by putting the maximum quasiparticle energy $E_{\max }=60 \mathrm{MeV}$ and the largest value of the orbital angular quantum number $l_{\max }=12$. The quasiparticle states in the continuum energy region are obtained with the box boundary condition $\phi\left(r_{\max }\right)=0$, and they are all discretized. All other details can be found in Ref. [44, 45].

We calculate the strength function $S_{\mathrm{Pad} 0}(\hbar \omega) \equiv \sum_{\nu}\left|\left\langle\nu\left|P_{00}^{\dagger}\right| 0\right\rangle\right|^{2} \delta\left(\hbar \omega-\hbar \omega_{\nu}\right)$ for the monopole $(L=0)$ pair-addition transfer and the strength function $S_{\operatorname{Prm} 0}(\hbar \omega) \equiv$ $\sum_{\nu}\left|\left\langle\nu\left|P_{00}\right| 0\right\rangle\right|^{2} \delta\left(\hbar \omega-\hbar \omega_{\nu}\right)$ for the pair-removal transfer, where, the pair-addition operator $P_{L M}^{\dagger}$ and pair-removal operator $P_{L M}$,

$$
\begin{aligned}
P_{L M}^{\dagger} & =\int d \boldsymbol{r} Y_{L M}(\hat{r}) \psi^{\dagger}(\boldsymbol{r} \downarrow) \psi^{\dagger}(\boldsymbol{r} \uparrow), \\
P_{L M} & =\int d \boldsymbol{r} Y_{L M}(\hat{r}) \psi(\boldsymbol{r} \uparrow) \psi(\boldsymbol{r} \downarrow),
\end{aligned}
$$

are creation and annihilation operators of the $S=0$ neutron pair with the total angular momentum $L$. These strength functions are evaluated using the solutions of the linear response equation (Eq. (11) [44]. Concerning the small imaginary part $i \epsilon$ appearing in the response function (Eq. (6) $)$, we choose $\epsilon=0.5 \mathrm{keV}$, which is much smaller than the value $\epsilon=50 \mathrm{keV}$ adopted in Ref.[45]. The QRPA eigen energies $\hbar \omega_{\nu}$ are then extracted by searching the peak energies of the pair-addition strength function $S_{\mathrm{Pad} 0}(\hbar \omega)$. The pairaddition strength

$$
B(\operatorname{Pad} 0 ; \mathrm{gs} \rightarrow \nu) \equiv\left|\left\langle 0\left|P_{00}\right| \nu\right\rangle\right|^{2}
$$

associated with individual QRPA eigen modes is evaluated by integrating the strength function $S_{\text {Pad0 }}(\hbar \omega)$ in an energy interval $\in\left[\hbar \omega_{\nu}-10 \epsilon, \hbar \omega_{\nu}+10 \epsilon\right]$. We also calculate the strength $B(\operatorname{Pad} 0 ; \mathrm{gs} \rightarrow \mathrm{gs})$ of the ground-state transfer (the pair rotation) as $B(\mathrm{Pad} 0 ; \mathrm{gs} \rightarrow \mathrm{gs})=$ $\left|\int r^{2} P_{\mathrm{gs}}^{(\mathrm{ad})}(r) d r\right|^{2}$ using the transition density $P_{\mathrm{gs}}^{(\mathrm{ad})}(r)$.

\section{VARIOUS COLLECTIVE PAIR-TRANSFER MODES IN SN ISOTOPES}

Figures 1(a) and 1(b) show the strength functions $S_{\mathrm{Pad} 0}(\hbar \omega)$ and $S_{\operatorname{Prm} 0}(\hbar \omega)$ for the monopole $(L=0)$ pair-addition and pair-removal transfers in neutron-rich ${ }^{134} \mathrm{Sn}$ and stable ${ }^{120} \mathrm{Sn}$, respectively. In Fig. 1] we also show results of the continuum QRPA calculation with solid curves. In this figure and only here, we used a slightly large value of the imaginary constant $\epsilon=50 \mathrm{keV}$ in order to make the peaks visible. It is noted here that most of the peaks seen in the pair-addition strength function $S_{\mathrm{Pad} 0}(\hbar \omega)$ in high energy region above the 

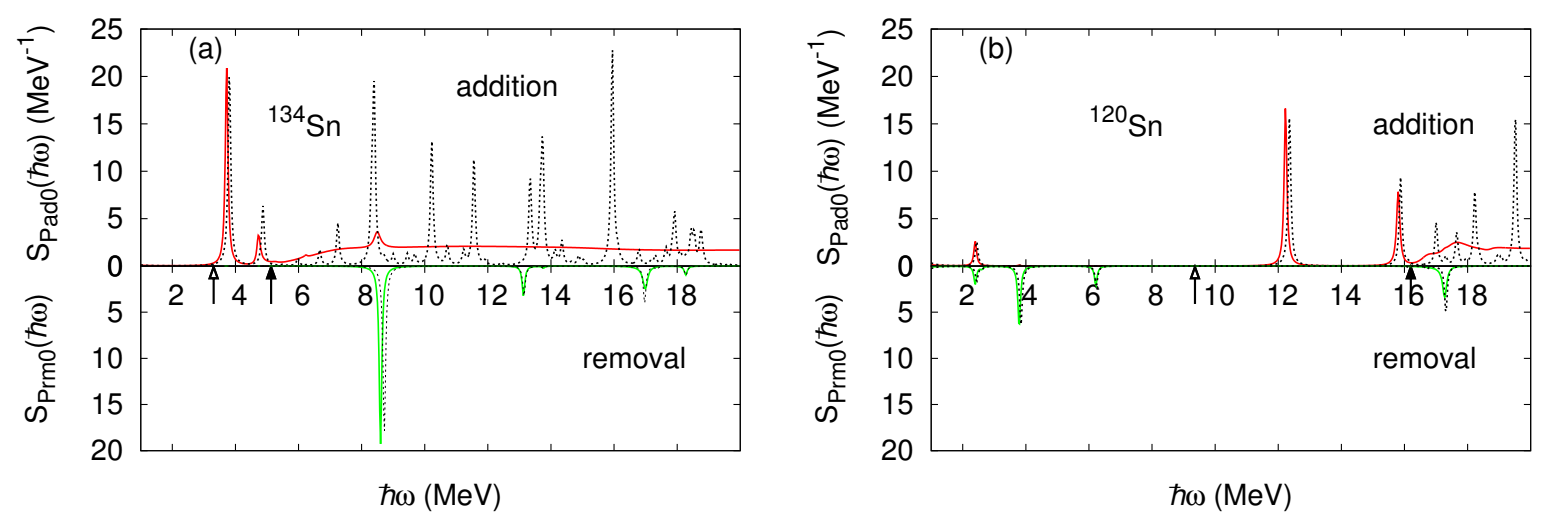

FIG. 1: (Color online) The strength function $S_{\text {Pad0 }}(\hbar \omega)$ for the pair-addition mode, plotted in the upper panel, and the strength function $S_{\operatorname{Prm} 0}(\hbar \omega)$ for the pair-removal mode (in the lower panel) in (a) ${ }^{134} \mathrm{Sn}$ and (b) ${ }^{120} \mathrm{Sn}$. The dotted curves are the results of the discretized QRPA while the solid curves are the result of the continuum QRPA calculation. The smoothing parameter $\epsilon=50$ $\mathrm{keV}$ is adopted in this figure. The arrows indicate the one- and the two-neutron separation energies $S_{1 n}=3.31 \mathrm{MeV}$ and $S_{2 n}=5.13 \mathrm{MeV}$ for ${ }^{134} \mathrm{Sn}, S_{1 n}=9.36 \mathrm{MeV}$ and $S_{2 n}=16.20 \mathrm{MeV}$ for ${ }^{120} \mathrm{Sn}$.

two neutron separation energy (the second arrow) are fictitious peaks originating from the discretization. We shall focus only on peaks for which the discretized and the continuum calculations give essentially the same peak energies and strengths.

First, we pay attention to the low-lying peaks at $2 \leq \hbar \omega \leq 4 \mathrm{MeV}$. As already discussed in Ref.[45], a prominent feature in neutron-rich ${ }^{134} \mathrm{Sn}$ is the large peak located at $\hbar \omega \approx 3.8$ $\mathrm{MeV}$ in the pair-addition strength function $S_{\mathrm{Pad} 0}(\hbar \omega)$. The pair-addition strength of this pair vibration is several times larger than that of the low-lying pair vibration at $\hbar \omega \approx 2.4$ $\mathrm{MeV}$ in stable ${ }^{120} \mathrm{Sn}$.

Let us next look at higher-lying pair-transfer modes up to $\hbar \omega=20 \mathrm{MeV}$, extending our previous study which covered only $\hbar \omega<10 \mathrm{MeV}$. There is no significant peak in the pair-addition strength $S_{\mathrm{Pad} 0}(\hbar \omega)$ in the high-frequency region in neutron-rich ${ }^{134} \mathrm{Sn}$ except the smooth strength distribution spread broadly above the two neutron separation energy. In stable ${ }^{120} \mathrm{Sn}$, on the other hand, two modes with large pair-addition strength appear at $\hbar \omega \approx 12.4 \mathrm{MeV}$ and $\hbar \omega \approx 15.9 \mathrm{MeV}$. They are located below the two-neutron separation energy $S_{2 n}=16.2 \mathrm{MeV}$. In the continuum QRPA calculation, these peaks have physical escaping widths since they are located above the one-neutron separation energy $S_{1 n}=9.36$ 


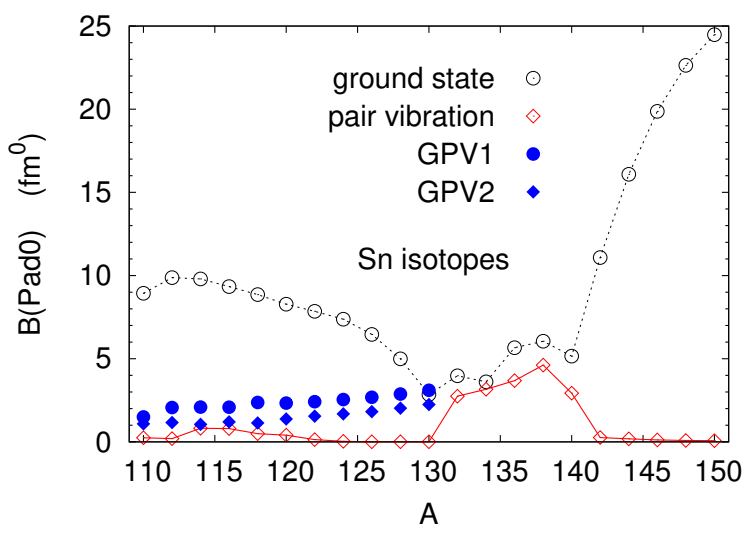

FIG. 2: (Color online) The neutron pair-addition strength $B(\operatorname{Pad} 0 ; g s \rightarrow \nu)$ for the giant pair vibrational modes GPV1 and GPV2, plotted with the filled circle and diamond, respectively, and the same quantity for the low-lying pair pair vibrational mode, plotted with open diamond, as well as the neutron pair-addition strength $B(\operatorname{Pad} 0 ; \mathrm{gs} \rightarrow \mathrm{gs})$ for the pair-rotational ground-state transfer (open circle), in even-even Sn isotopes.

$\mathrm{MeV}$, but their widths are actually very small. As we show later, these two peaks can be regarded as so called giant pair vibration (GPV) [55 57]. The GPV is a collective pairtransfer mode of adding or removing two neutrons in the next major shell beyond the valence shell. In the present calculation, the giant pair vibration consists of two peaks. We call these two peaks GPV1 $(\hbar \omega \approx 12.4 \mathrm{MeV})$ and GPV2 $(\hbar \omega \approx 15.9 \mathrm{MeV})$, and we discuss them in detail in Section V. The giant pair vibrations exist in all the isotopes with $110 \lesssim A<132$.

In the pair-removal strength function $S_{\mathrm{Prm} 0}(\hbar \omega)$ in ${ }^{134} \mathrm{Sn}$ (Fig. 1(a)) there exist a giant pair-removal vibration with large strength at $\hbar \omega \approx 8.7 \mathrm{MeV}$. It corresponds to the neutron pair-removal from the $N=50-82$ shell[58]. In this paper, however, we do not investigate the pair-removal modes.

In Fig. 2, we show the systematical behavior of the pair-additional strength $B(\operatorname{Pad} 0 ;$ gs $\rightarrow$ $\nu)$ for the two giant pair vibrations GPV1 and GPV2, as well as the strength $B(\operatorname{Pad} 0$; gs $\rightarrow \nu)$ of the low-lying pair vibration, and the strength $B$ (Pad0;gs $\rightarrow$ gs) for the pair-rotational ground-state transfer in the $\mathrm{Sn}$ isotopes $A=110-150$. It is seen that the pair-addition strengths of GPV1 and GPV2 increase with the neutron number, and at $A \sim 130$ they become comparable to those of the characteristic low-lying pair vibrations and those of the 


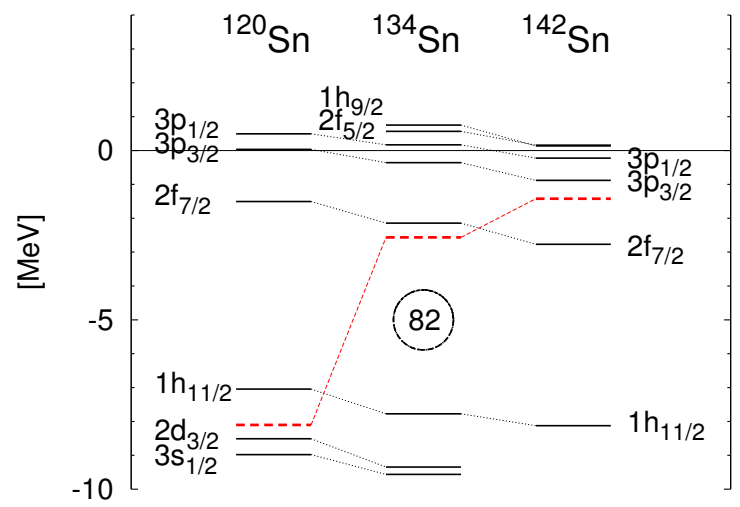

FIG. 3: (Color online) The Hartree-Fock single-particle energies $e_{\mathrm{HF}}$ of neutrons in ${ }^{120} \mathrm{Sn},{ }^{134} \mathrm{Sn}$ and ${ }^{142} \mathrm{Sn}$. The dashed lines show the neutron Fermi energies $\lambda_{n}=-8.10,-2.56$ and $-1.42 \mathrm{MeV}$ for the three isotopes.

ground-state transfer in ${ }^{132-140} \mathrm{Sn}$.

The values of the pair-addition strength and the excitation energy for the giant pair vibrational states are also listed in Table III and IV.

\section{MICROSCOPIC STRUCTURES OF LOW-LYING PAIR VIBRATIONAL MODES}

\section{A. Dominant two-quasiparticle configurations}

In Ref.[45], we have discussed the collectivity of the low-lying pair-addition vibration $\left(\hbar \omega_{\nu}=3.81 \mathrm{MeV}\right)$ in ${ }^{134} \mathrm{Sn}$ by comparing its strength $B(\operatorname{Pad} 0)=3.23$ with the singleparticle strength $B_{\text {s.p. }}(\operatorname{Pad} 0)=(2 j+1) / 8 \pi=0.18$ and 0.15 , evaluated for pure independent two-neutron configurations $\left[2 f_{7 / 2}\right]^{2}$ and $\left[3 p_{3 / 2}\right]^{2}$, respectively. We observe a large collective enhancement of a factor of more than ten. In this section, we reveal the origin of this collectivity by examining microscopic structure of this mode.

First we evaluate and analyze the forward and backward amplitudes $X_{i i^{\prime}}^{\nu}$ and $Y_{i i^{\prime}}^{\nu}$ for the two-quasiparticle configurations, given by Eqs.(18) and (19), respectively. We find that the two-neutron transfer modes discussed above almost all the amplitudes ( $\gtrsim 99 \%)$ are exhausted by neutron two-quasiparticle states. In the following, we discuss only the neutron amplitudes. 


\begin{tabular}{ccccc}
\hline \hline & & $E_{i}+E_{i^{\prime}}$ & $X_{i i^{\prime}}^{\nu}$ & $Y_{i i^{\prime}}^{\nu}$ \\
\hline${ }^{134} \mathrm{Sn}$ & {$\left[3 p_{3 / 2}\right]^{2}$} & 4.49 & 0.752 & 0.0006 \\
$\hbar \omega_{\nu}=3.81 \mathrm{MeV}$ & {$\left[1 h_{9 / 2}\right]^{2}$} & 6.71 & -0.368 & -0.0017 \\
& {$\left[2 f_{7 / 2}\right]^{2}$} & 1.50 & -0.297 & -0.0325 \\
& {$\left[2 f_{5 / 2}\right]^{2}$} & 6.35 & -0.279 & -0.0013 \\
& {$\left[1 i_{13 / 2}\right]^{2}$} & 9.61 & 0.241 & 0.0021 \\
& {$\left[3 p_{1 / 2}\right]^{2}$} & 5.52 & 0.177 & -0.0005 \\
$\hbar \omega_{\nu}=2.44 \mathrm{MeV}$ & {$\left[3 p_{3 / 2}\right]\left[4 p_{3 / 2}\right]$} & 6.78 & 0.101 & -0.0004 \\
\hline${ }^{120} \mathrm{Sn}$ & {$\left[1 h_{11 / 2}\right]^{2}$} & 3.60 & 0.659 & -0.059 \\
& {$\left[2 d_{3 / 2}\right]^{2}$} & 2.52 & 0.606 & 0.014 \\
& {$\left[3 s_{1 / 2}\right]^{2}$} & 2.85 & -0.436 & -0.021 \\
& {$\left[1 g_{7 / 2}\right]^{2}$} & 4.80 & 0.087 & -0.072 \\
& {$\left[2 d_{5 / 2}\right]^{2}$} & 6.76 & -0.079 & 0.039 \\
\hline \hline
\end{tabular}

TABLE I: The forward amplitude $X_{i i^{\prime}}^{\nu}$ and the backward amplitude $Y_{i i^{\prime}}^{\nu}$ for the low-lying pair vibration modes in ${ }^{134} \mathrm{Sn}\left(\hbar \omega_{\nu}=3.81 \mathrm{MeV}\right)$ and in ${ }^{120} \mathrm{Sn}\left(\hbar \omega_{\nu}=2.44 \mathrm{MeV}\right)$. The two-quasiparticle configurations which have the largest values of $\left|X_{i i^{\prime}}^{\nu}\right|$ are listed.

Let us consider the pair vibration at $\hbar \omega_{\nu}=3.81 \mathrm{MeV}$ in neutron-rich ${ }^{134} \mathrm{Sn}$. Table 【lists the two-quasiparticle components having the largest absolute values of the forward amplitude $\left|X_{i i^{\prime}}^{\nu}\right|$. The component with the largest amplitude is $\left[3 p_{3 / 2}\right]^{2}$ with $X_{i i^{\prime}}^{\nu}=0.752$. There are seven components whose $X$-amplitudes satisfy $\left|X_{i i^{\prime}}^{\nu}\right|>0.1 ;\left[3 p_{3 / 2}\right]^{2},\left[1 h_{9 / 2}\right]^{2},\left[2 f_{7 / 2}\right]^{2},\left[2 f_{5 / 2}\right]^{2}$ $\left[1 i_{13 / 2}\right]^{2}$ and $\left[3 p_{1 / 2}\right]^{2}$. The main components indicate that the low-lying pair vibrational state in ${ }^{134} \mathrm{Sn}$ has some degree of collectivity. The Hartree-Fock(HF) single-particle energies of neutrons are shown in Fig. 3. The quasiparticle orbits of the main components are those located near the Fermi energy, all of which are either weakly bound or unbound resonant quasiparticle states.

In the case of the low-lying pair vibration $\left(\hbar \omega_{\nu}=2.44 \mathrm{MeV}\right)$ in stable ${ }^{120} \mathrm{Sn}$, there are only three components $\left[1 h_{11 / 2}\right]^{2},\left[2 d_{3 / 2}\right]^{2}$ and $\left[3 s_{1 / 2}\right]^{2}$, having large forward amplitudes satisfying $\left|X_{i i^{\prime}}^{\nu}\right|>0.1$, see Table I. It is evident that the collectivity is smaller here than that of the pair vibration in ${ }^{134} \mathrm{Sn}$. This is consistent qualitatively with a much smaller value of the 

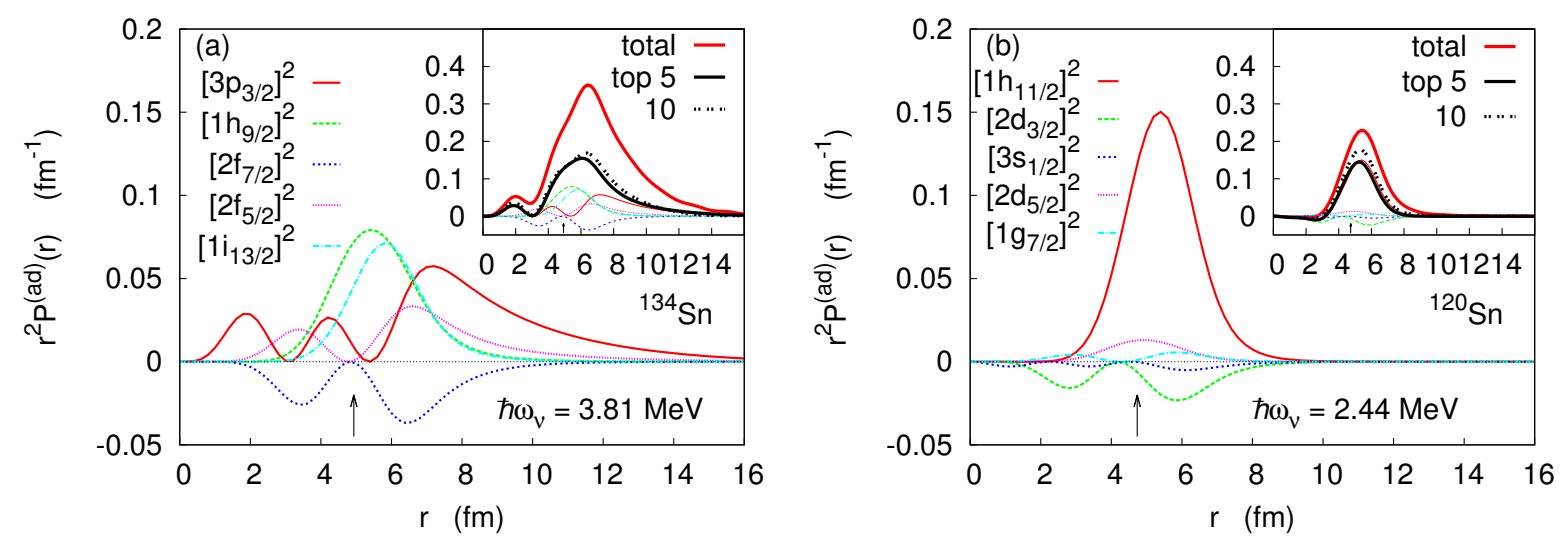

FIG. 4: (Color online) (a) The decomposed transition densities $r^{2} P_{\nu L, i i^{\prime}}^{(\mathrm{ad})}(r)$ for the largest twoquasiparticle components of the low-lying pair vibration mode $\left(\hbar \omega_{\nu}=3.81 \mathrm{MeV}\right)$ in ${ }^{134} \mathrm{Sn}$ (cf. Table I). In the inset, shown also are the total transition density $r^{2} P_{\nu L}^{(\mathrm{ad})}(r)$ and partial sums of the decomposed transition densities of the largest five and ten two-quasiparticle components. (b) The same as (a), but for the low-lying pair vibration mode $\left(\hbar \omega_{\nu}=2.44 \mathrm{MeV}\right)$ in ${ }^{120} \mathrm{Sn}$. The arrow indicates the neutron rms radius $R_{N, r m s}\left(=\sqrt{\left\langle r_{n}^{2}\right\rangle}\right)=4.93 \mathrm{fm}$ and $4.73 \mathrm{fm}$ for ${ }^{134} \mathrm{Sn}$ and ${ }^{120} \mathrm{Sn}$, respectively.

pair-addition strength $B(\operatorname{Pad} 0)=0.40$ in ${ }^{120} \mathrm{Sn}$ than $B(\operatorname{Pad} 0)=3.16$ in ${ }^{134} \mathrm{Sn}$.

Next, we analyze the transition density $P_{\nu L}^{(\mathrm{ad})}(r)$ by looking into the decomposed transition densities $P_{\nu L, i i^{\prime}}^{(\mathrm{ad})}(r)$ (Eq.(29) ) associated with the main two-quasiparticle configurations. They are shown in Figs. 4(a) and 4(b), for the low-lying pair vibration in ${ }^{134} \mathrm{Sn}$ and ${ }^{120} \mathrm{Sn}$, respectively.

We see in Fig. 4(a) the following characteristics of the pair vibration in ${ }^{134} \mathrm{Sn}$. The individual decomposed transition densities have much smaller amplitudes than that of the total transition density. Even if we sum the transition densities of the largest five components (the thick solid curve in the inset), it accounts for only approximately half of the total transition density. The situation is essentially the same also for a sum of the largest ten components (the thick dotted curve). We note also that the tail of the total transition density extending to $r \sim 16 \mathrm{fm}$ is not reproduced by the largest five (ten) components. The component $\left[3 p_{3 / 2}\right]^{2}$ has the largest tail among the five (note that $3 p_{3 / 2}$ is weakly bound single-particle orbit with low angular momentum), but its tail amplitude is quite smaller than that of total transition density. We thus find that contributions from small components 
other than the largest five or ten play important role in some way to produce the large pairaddition strength $B(\operatorname{Pad} 0)$ and the characteristic transition density of the low-lying pair vibration in ${ }^{134} \mathrm{Sn}$.

The situation of the low-lying pair vibration in ${ }^{120} \mathrm{Sn}$ is different. As shown in Fig. 4(b), the $\left[1 h_{11 / 2}\right]^{2}$ component has a dominant contribution to the pair-addition transition density, amounting more than two-thirds of the total transition density around the surface. Because the HF single-particle energy of the $1 h_{11 / 2}$ orbit in ${ }^{120} \mathrm{Sn}$ is located deeply at $e_{1 h_{11 / 2}}=-7.00$ $\mathrm{MeV}$, and the Fermi energy is also deep $\lambda_{n}=-8.10 \mathrm{MeV}$ (cf. Fig. [3), the transition density of the component $\left[1 h_{11 / 2}\right]^{2}$ as well as the total do not extend far outside the surface.

\section{B. High- $l$ two-quasiparticle configurations and di-neutron correlation}

We have seen in the previous section that the main components of the pair vibration mode in ${ }^{134} \mathrm{Sn}$ reproduces only about a half of the total transition density. In this subsection we investigate how other small two-quasiparticle components contribute.

For this purpose, we put all the two-quasiparticle configurations into subgroups which are specified with the orbital angular momentum $l$ of the quasiparticle states. Note that we have only two-quasiparticle combinations $i i^{\prime}=[n l j]\left[n^{\prime} l^{\prime} j^{\prime}\right]$ satisfying $l=l^{\prime}$ and $j=j^{\prime}$ for $0^{+}$modes. We then calculate a partial sum of the decomposed transition densities $P_{\nu L, l_{c u t}}^{(\mathrm{ad})}(r)=\sum_{i i^{\prime}, l \leq l_{c u t}} P_{\nu L, i i^{\prime}}^{(\mathrm{ad})}(r)$, where the orbital angular momenta $l$ are taken into account up to a cut-off values $l_{\text {cut }}$. Figures 5(a) and 5(b) show the $l$-cutted transition densities $P_{\nu L, l_{c u t}}^{(\mathrm{ad})}(r)$ with $l_{\text {cut }}=0,1,2, \cdots, 12$ for low-lying pair-addition vibration in neutron-rich ${ }^{134} \mathrm{Sn}$ and in stable ${ }^{120} \mathrm{Sn}$, respectively.

Let us first look at the case of ${ }^{134} \mathrm{Sn}$, i.e. Fig. 5(a). It is clearly seen that a large number of the orbital angular momenta covering up to the maximum value $l_{\max }=12$ have non-negligible contributions, especially at larger values of $r$. The highest orbital angular momentum of the occupied HF single-particle orbits in ${ }^{134} \mathrm{Sn}$ is $l_{\text {occ }}=5\left(1 h_{11 / 2}\right)$. The sum up to $l_{\text {cut }}=l_{\text {occ }}=5$ can account for only about a half of the total transition density. The other half comes from the contributions of quasiparticle states with higher orbital angular momenta $l>5$, which are all unbound continuum states. A remarkable feature is that at large distances, $r \gtrsim 8 \mathrm{fm}$, contributions from different l's are similar in magnitude, depending only weakly on $l$, and their contributions are accumulated coherently to build up the total transition density of 

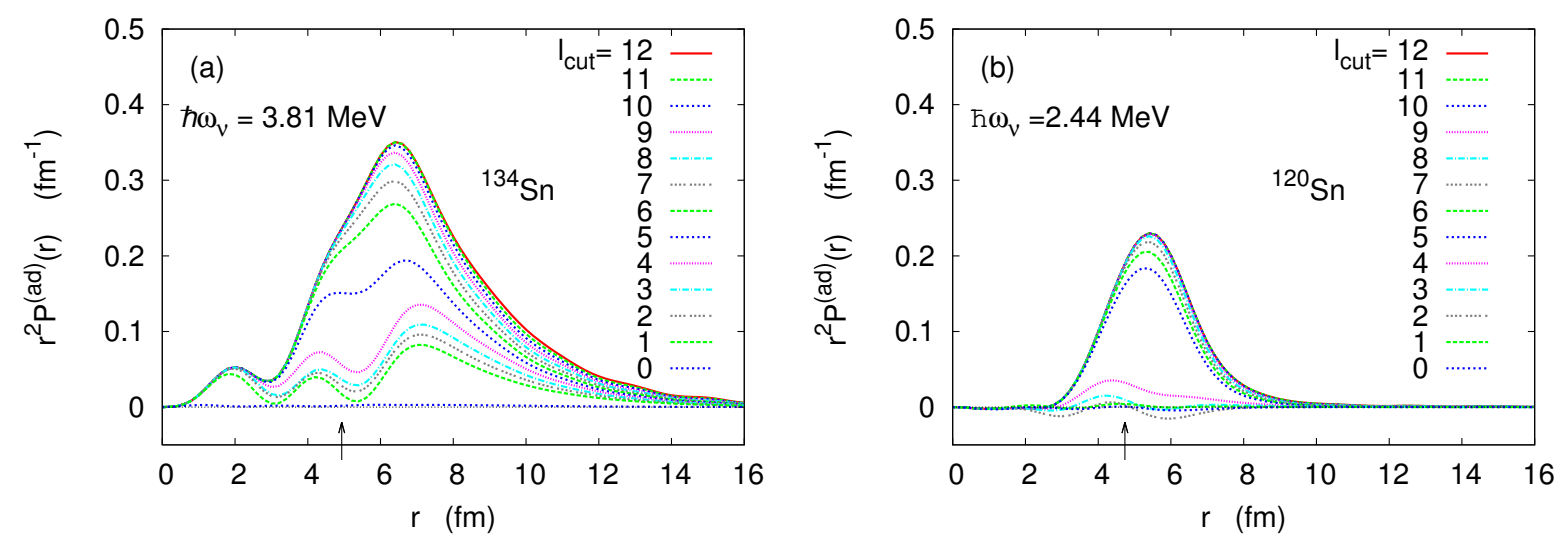

FIG. 5: (Color online) The $l$-cutted transition densities $r^{2} P_{\nu L, l_{c u t}}^{(\mathrm{ad})}(r)$ with $l_{\text {cut }}=0,1,2, \cdots, 12$ for the low-lying pair vibration modes (a) in ${ }^{134} \mathrm{Sn}$ and (b) in ${ }^{120} \mathrm{Sn}$. The arrow indicates the neutron rms radius $R_{N, r m s}$.

the pair-addition vibration.

The coherent contribution up to high orbital angular momenta is a signature of the di-neutron correlation. Let us recall that a two-particle wave function made of the $J=$ 0-coupled single-particle states brings about an angular correlation $\sum_{m} Y_{l m}^{*}\left(\hat{\boldsymbol{r}}_{1}\right) Y_{l m}\left(\hat{\boldsymbol{r}}_{2}\right) \sim$ $P_{l}\left(\cos \theta_{12}\right)$ with respect to the relative angle $\theta_{12}$ between the positions $\hat{\boldsymbol{r}}_{1}$ and $\hat{\boldsymbol{r}}_{2}$ of the two particles. Since $P_{l}\left(\cos \theta_{12}\right)$ is peaked at $\theta_{12}=0$ and always positive for $\theta_{12} \lesssim 1 / l$, and if we superpose them coherently over a large number of $l$ in a range $0 \leq l \lesssim l_{\text {corr }}$, the obtained two-particle wave function may exhibit an angular correlation at small relative angles $\theta_{12} \lesssim 1 / l_{\text {corr }}$. The validity of this argument is confirmed for the Cooper pair wave function and the pair density $\tilde{\rho}(r)$ in the HFB ground state[19, 20]. Extending this argument to the pair-addition transition density $P_{L \nu}^{(\mathrm{ad})}(r)$, we deduce that the coherent contribution up to high orbital angular momenta suggests the di-neutron correlation, a spatial correlation at short distances in the pair-addition transition density[28]. In other words, it suggests transfer of "a di-neutron" in exciting the low-lying pair vibrational mode in ${ }^{134} \mathrm{Sn}$.

Concerning the pair vibration in ${ }^{120} \mathrm{Sn}$, Fig. 5(b), we see some coherent contributions of high- $l$ quasiparticles with $5<l \lesssim 8-9$, but it is to much lesser extent than that in ${ }^{134} \mathrm{Sn}$. The difference is very clear for the tail region $r \gtrsim 8 \mathrm{fm}$; in ${ }^{134} \mathrm{Sn}$ the coherent high- $l$ contribution is evident while in ${ }^{120} \mathrm{Sn}$ the transition density itself is vanishing. 


\begin{tabular}{cccrr}
\hline \hline & & $E_{i}+E_{i^{\prime}}$ & $X_{i i^{\prime}}^{\nu}$ & $Y_{i i^{\prime}}^{\nu}$ \\
\hline${ }^{120} \mathrm{Sn}$ & {$\left[2 f_{7 / 2}\right]^{2}$} & 13.53 & 0.936 & 0.0005 \\
$\hbar \omega_{\nu}=12.36 \mathrm{MeV}$ & {$\left[1 h_{9 / 2}\right]^{2}$} & 19.77 & -0.151 & -0.0006 \\
GPV1 & {$\left[1 i_{13 / 2}\right]^{2}$} & 22.41 & 0.145 & 0.0007 \\
& {$\left[2 f_{5 / 2}\right]^{2}$} & 18.61 & -0.122 & -0.0003 \\
& {$\left[3 p_{3 / 2}\right]^{2}$} & 16.27 & 0.116 & 0.0002 \\
$\hbar \omega_{\nu}=15.88 \mathrm{MeV}$ & {$\left[1 h 11_{3 / 2}\right]^{2}$} & 3.60 & -0.114 & 0.0129 \\
\cline { 2 - 5 } $\mathrm{GPV} 2$ & {$\left[3 p_{3 / 2}\right]^{2}$} & 16.27 & 0.877 & 0.0003 \\
& {$\left[2 f_{7 / 2}\right]^{2}$} & 13.53 & -0.228 & 0.0015 \\
& {$\left[3 p_{1 / 2}\right]^{2}$} & 17.14 & -0.156 & -0.0001 \\
& {$\left[1 h_{9 / 2}\right]^{2}$} & 19.77 & -0.154 & -0.0019 \\
& {$\left[2 f_{5 / 2}\right]^{2}$} & 18.61 & -0.154 & -0.0006 \\
& {$\left[1 i_{13 / 2}\right]^{2}$} & 22.41 & 0.121 & -0.0023 \\
\hline \hline
\end{tabular}

TABLE II: The forward amplitudes $X_{i i^{\prime}}^{\nu}$ and the backward amplitudes $Y_{i i^{\prime}}^{\nu}$ for the giant pair vibrations, GPV1 $\left(\hbar \omega_{\nu}=12.36 \mathrm{MeV}\right)$ and GPV2 $\left(\hbar \omega_{\nu}=15.88 \mathrm{MeV}\right)$, in ${ }^{120} \mathrm{Sn}$. The largest two-quasiparticle components satisfying $\left|X_{i i^{\prime}}^{\nu}\right|>0.1$ are listed.

\section{GIANT PAIR VIBRATIONS IN $A<132$ ISOTOPES}

Let us analyze the high-lying pair-addition modes GPV2 at $\hbar \omega_{\nu}=15.88 \mathrm{MeV}$ and GPV1 at $\hbar \omega_{\nu}=12.36 \mathrm{MeV}$ in ${ }^{120} \mathrm{Sn}$ (cf. Fig. 1(b)).

We first note that the pair-addition strengths of these modes, $B(\operatorname{Pad} 0)=1.374$ of GPV2 and $B(\operatorname{Pad} 0)=2.332$ of GPV1, are about eight times larger than the single-particle values $B_{\text {s.p. }}(\operatorname{Pad} 0)=0.16$ for $j=3 / 2$ and $B_{\text {s.p. }}(\operatorname{Pad} 0)=0.32$ for $j=7 / 2$, respectively.

Let us first examine the phonon amplitudes. Table【shows the forward amplitudes $X_{i i^{\prime}}^{\nu}$ of the dominant two-quasiparticle components of GPV1 and GPV2. Here the two-quasiparticle configurations with large amplitudes $\left|X_{i i^{\prime}}^{\nu}\right|>0.1$ are plotted. All these dominant components, except $\left[2 d_{5 / 2}\right]\left[4 d_{5 / 2}\right]$, are made of the quasiparticle states $\left[2 f_{7 / 2}\right],\left[3 p_{3 / 2}\right],\left[3 p_{1 / 2}\right],\left[1 h_{9 / 2}\right]$, $\left[2 f_{5 / 2}\right]$ and $\left[1 i_{13 / 2}\right]$, which all belong to the shell next to the valence shell, i.e. the one above the $N=82$ shell gap (cf. Fig. 3). This confirms that GPV1 and GPV2 are indeed the giant 
pair vibrations. (Note that the two-quasiparticle configuration $\left[2 d_{5 / 2}\right]\left[4 d_{5 / 2}\right]$ is a particle-hole excitation from the bound $2 d_{5 / 2}$ orbit to a discretized continuum state in the partial wave $d_{5 / 2}$. This component contributes very little to the pair-transfer mode.) Even though the largest components $\left[2 f_{7 / 2}\right]^{2}$ in GPV1 and $\left[3 p_{3 / 2}\right]^{2}$ in GPV2 have predominant amplitudes $X_{i i^{\prime}}^{\nu}=0.94$ and 0.88 , respectively, the number ( $\sim$ seven) of large components which satisfy $\left|X_{i i^{\prime}}^{\nu}\right|>0.1$ show the degree of collectivity similar to that of the pair vibration in ${ }^{134} \mathrm{Sn}$.

Figures 6(a) and 6(b) show the transition densities $P_{\nu L}^{(\mathrm{ad})}(r)$ of the GPV2 and GPV1 modes, respectively. It is seen that the transition densities extend to far outside the nuclear surface, reaching $r \sim 16$ and $r \sim 14 \mathrm{fm}$, respectively. Plotted are also the decomposed transition densities $P_{\nu L, i i^{\prime}}^{(\mathrm{ad})}(r)$ for the largest five components listed in Table II. In the GPV2 mode (see the inset of Fig. 6(a)), the amplitude of transition density of the main component $\left[3 p_{3 / 2}\right]^{2}$ is significantly smaller than that of the total transition density. Even if we consider the superposition of the decomposed transition densities with the largest five components, it reproduces only one-quarter of the total transition density of the GPV2 mode. A similar situation is observed also in the case of GPV1 (Fig. 6(b)), where the most dominant component $\left[2 f_{7 / 2}\right]^{2}$ accounts for only one-third of the maximum of the total transition density, and a sum of the largest five components give only a bit more than a half. The large components alone are not sufficient to account for the collectivity of GPV1 and GPV2, especially that of GPV2.

We also analyze the microscopic structure of these modes in terms of the orbital angular momentum $l$ of the quasiparticle orbits. The method is the same as what we have done for the low-lying pair vibration, i.e. we plot partial sums of the decomposed transition densities $P_{\nu L, l_{c u t}}^{(\mathrm{ad})}(r)=\sum_{i i^{\prime}, l \leq l_{c u t}} P_{\nu L, i i^{\prime}}^{(\mathrm{ad})}(r)$, for various values of the angular momentum cut-off $l_{c u t}$. The results are shown in Fig. 7.

It is seen in both Figs. 7 (a) and 7(b) that two-quasiparticle components with different $l$ 's contribute coherently, and the contributions from high- $l$ states (with $l>l_{\text {occ }}=5$ ) are significant. The latter feature is very strong in the external region $r \gtrsim 8 \mathrm{fm}$ of GPV2 (Fig. 7(a)). This is quite similar to the features observed for the low-lying pair vibration in ${ }^{134} \mathrm{Sn}$ (Fig. 5(a)). Applying the same arguments on Fig. 5(a), we deduce that the di-neutron correlation appears also in the GPV2 mode in the stable ${ }^{120} \mathrm{Sn}$ isotope, and that the GPV1 mode also exhibits the same feature, but to a lesser extent. This is in contrast to the lowlying pair vibration in ${ }^{120} \mathrm{Sn}$ (Fig. 5(b)), where the di-neutron feature is seen only weakly. 

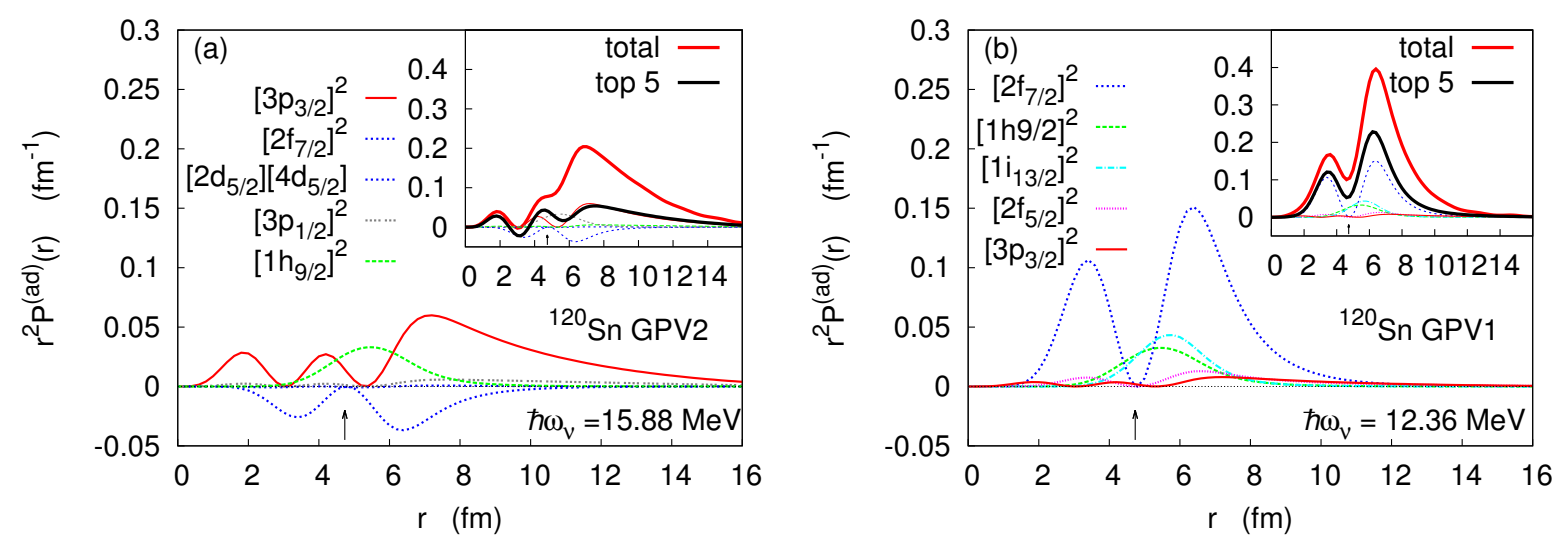

FIG. 6: (Color online) (a) The decomposed transition densities $r^{2} P_{\nu L, i i^{\prime}}^{(\mathrm{ad})}(r)$ for the largest twoquasiparticle components of the giant pair vibration GPV2 at $\hbar \omega_{\nu}=15.88 \mathrm{MeV}$ in ${ }^{120} \mathrm{Sn}$ (cf. Table II). In the inset, shown also are the total transition density $r^{2} P_{\nu L}^{(\mathrm{ad})}(r)$ and a partial sum of the decomposed transition densities of the largest five two-quasiparticle components. The arrow indicates the neutron rms radius $R_{N, r m s}=4.73 \mathrm{fm}$. (b) The same as (a) but for the giant pair vibration GPV1 at $\hbar \omega_{\nu}=12.36 \mathrm{MeV}$ in ${ }^{120} \mathrm{Sn}$.
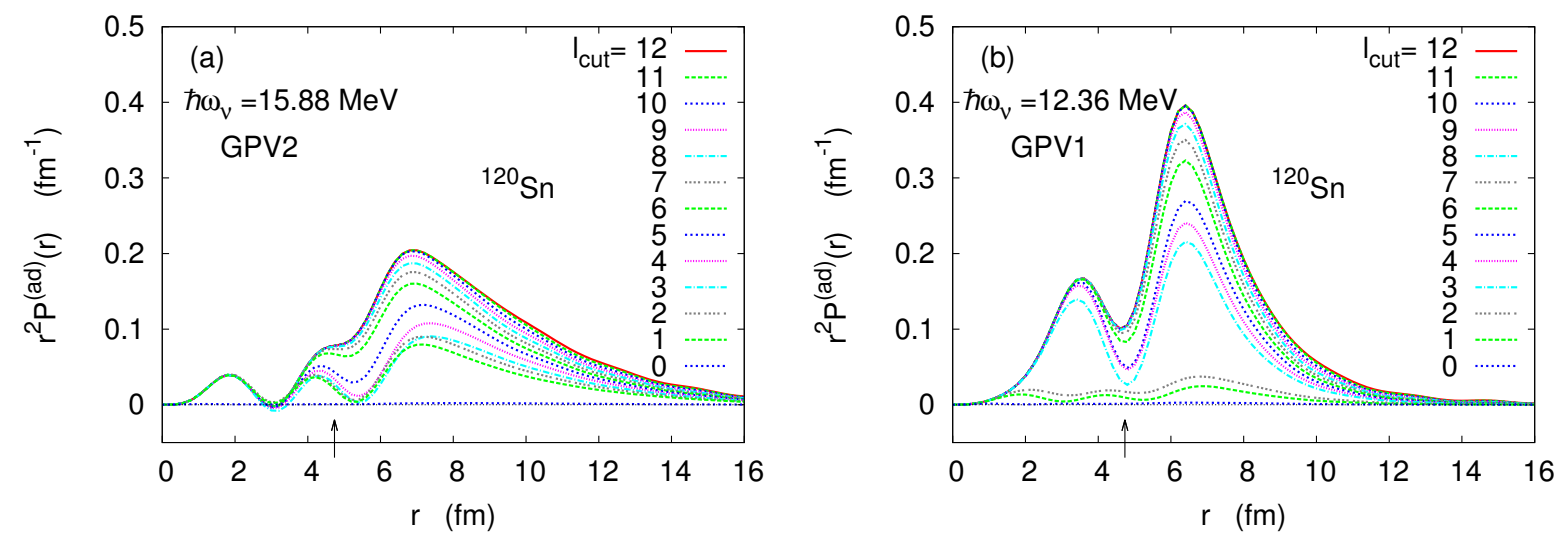

FIG. 7: (Color online) The $l$-cutted transition densities $r^{2} P_{\nu L, l_{c u t}}^{(\mathrm{ad})}(r)$ with $l_{\text {cut }}=0,1,2, \cdots, 12$ for the giant pair vibrations (a) GPV2 $\left(\hbar \omega_{\nu}=15.88 \mathrm{MeV}\right)$ and (b) GPV1 $\left(\hbar \omega_{\nu}=12.36 \mathrm{MeV}\right)$ in ${ }^{120} \mathrm{Sn}$. The arrow indicates the neutron rms radius $R_{N, r m s}$.

\section{PAIR ROTATION}

In this section we show the microscopic structure of the pair rotation, i.e. the transfer mode populating the ground state of neighboring $N+2$ isotope.

We decompose the transition density $P_{\mathrm{gs}}^{(\mathrm{ad})}(r)$ with respect to the quasiparticle states 

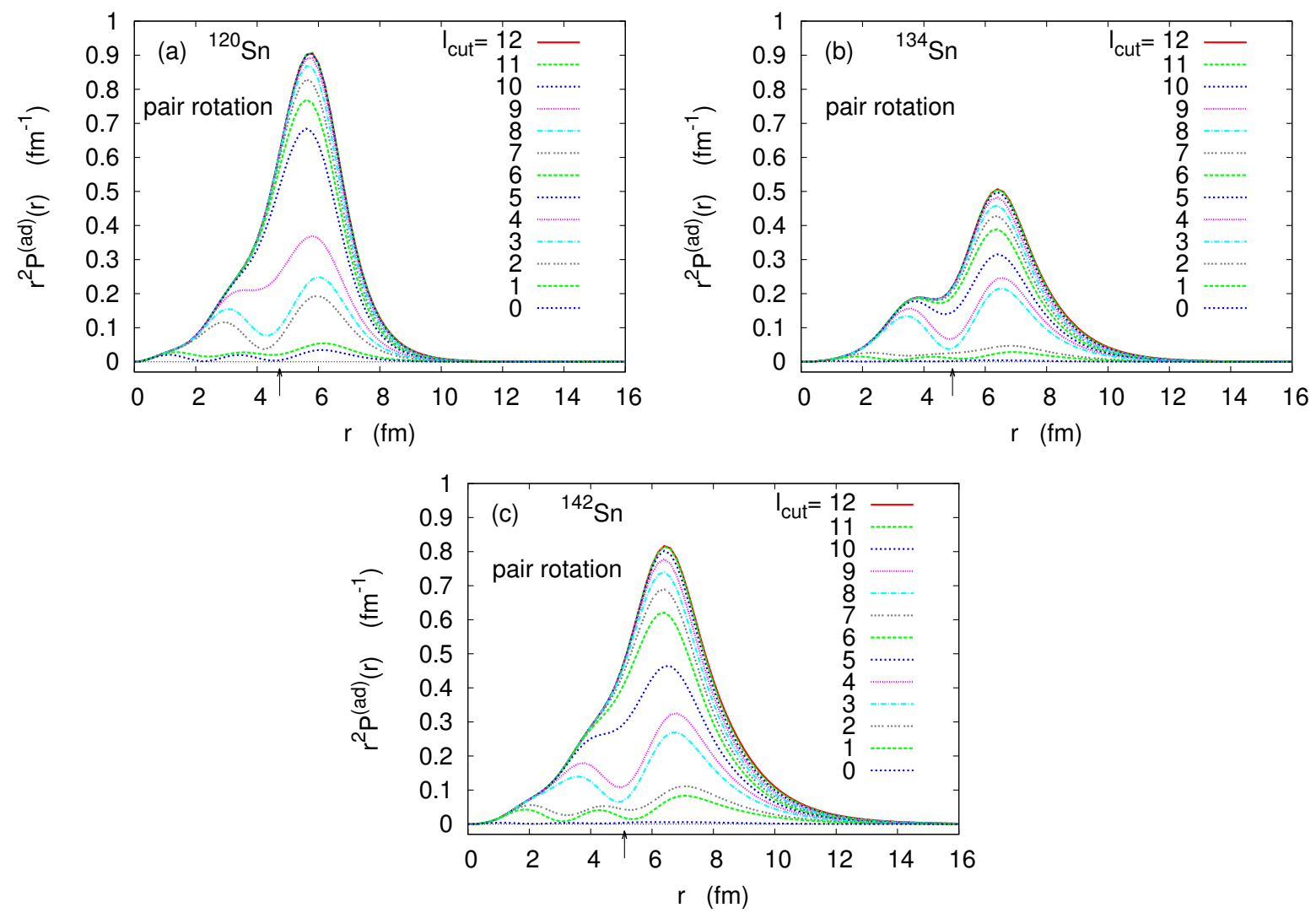

FIG. 8: (Color online) The $l$-cutted transition densities $r^{2} P_{\mathrm{gs}, l_{\text {cut }}}^{(\mathrm{ad})}(r)$ with $l_{\text {cut }}=0,1,2, \cdots, 12$ for the pair rotational modes in (a) ${ }^{120} \mathrm{Sn}$, (b) ${ }^{134} \mathrm{Sn}$ and (c) ${ }^{142} \mathrm{Sn}$. The arrow indicates the neutron rms radius $R_{N, r m s}=4.73 \mathrm{fm}, 4.93 \mathrm{fm}$ and $5.10 \mathrm{fm}$ for ${ }^{120} \mathrm{Sn},{ }^{134} \mathrm{Sn}$ and ${ }^{142} \mathrm{Sn}$, respectively.

involved, as is shown in Eqs.(33) and (34). We then introduce a partial sum specified by the orbital angular momentum cutoff $l_{\text {cut }}$ as $P_{\mathrm{gs}, l_{\text {cut }}}^{(\mathrm{ad})}(r)=\sum_{i, l<l_{\text {cut }}} P_{\mathrm{gs}, i i}^{(\mathrm{ad})}(r)$ (cf Eq.(34)). This decomposition is shown in Fig. 8(a)-(c) for the ground-state transfer of ${ }^{120} \mathrm{Sn},{ }^{134} \mathrm{Sn}$ and ${ }^{142} \mathrm{Sn}$, respectively.

The amplitude in the external region develops significantly with increasing the neutron number, from ${ }^{120} \mathrm{Sn}$ to ${ }^{134} \mathrm{Sn}$ and ${ }^{142} \mathrm{Sn}$. In addition to this overall feature (discussed already in Ref.[45]), we find here that the high- $l$ contribution (with $l \geq 5$ ) to the transition density grows with increasing the neutron number. Looking at $r=6 \mathrm{fm}$ where the plotted transition density is close to the largest, the high- $l$ contribution is $\sim 20 \%, \sim 30 \%$ and $\sim 40 \%$ in ${ }^{120} \mathrm{Sn}$, ${ }^{134} \mathrm{Sn}$ and ${ }^{142} \mathrm{Sn}$, respectively. It is also obvious that all high-l's contribute coherently to build up the pair-addition transition density $P_{\mathrm{gs}}^{(\mathrm{ad})}(r)$. In short, the features suggesting the di-neutron correlation are seen well in the pair rotational mode in ${ }^{134} \mathrm{Sn}$, and especially in 
${ }^{142} \mathrm{Sn}$, but not very significantly in ${ }^{120} \mathrm{Sn}$.

It is noted here that the Fermi energies in ${ }^{134} \mathrm{Sn}$ and ${ }^{142} \mathrm{Sn}$ are small $\left(\lambda_{n}=-2.56\right.$ and $-1.42 \mathrm{MeV}$, respectively), and the lowest energy quasiparticle states in these isotopes are $3 p_{1 / 2}, 3 p_{3 / 2}$ and $2 f_{7 / 2}$ states (cf Fig. 3). However, the contribution of these quasiparticle states to the pair-addition transition density is not very large as seen in the curve with $l_{\text {cut }}=3$. The high- $l$ quasiparticle states, which give dominant part of the transition density in the external region, are all unbound quasiparticle states in the continuum energy region. This is one of the mechanisms that make the transition density very extended in ${ }^{134} \mathrm{Sn}$ and ${ }^{142} \mathrm{Sn}$.

All the above mentioned features of the pair rotation in ${ }^{134} \mathrm{Sn}$ and ${ }^{142} \mathrm{Sn}$ have some similarity to those of the low-lying pair vibration in ${ }^{134} \mathrm{Sn}$ and the GPV's in the isotopes with $A<132$, discussed in the previous sections.

\section{SYSTEMATICS}

In ${ }^{110-130} \mathrm{Sn}$, we systematically observe two peaks of the high-lying giant pair vibrations, GPV1 and GPV2. In order to clarify the isotopic trends of the low-lying pair vibration, the giant pair vibration and the pair rotation, we have performed systematic numerical calculations for all the even- $N$ isotopes from $A=120$ to $A=150$. In ${ }^{132}$ Sn where the $N=82$ shell is closed and the neutron pair gap vanishes, we find two low-lying pair vibration in the pair-addition channel. The lowest one, denoted PV1 hereafter, can be regarded as a pair-addition mode populating the ground state of ${ }^{134} \mathrm{Sn}$, while the second one, denoted $\mathrm{PV} 2$, is another pair-addition mode populating the second $0^{+}$state in ${ }^{134} \mathrm{Sn}$. In ${ }^{134-140} \mathrm{Sn}$ we find the characteristic low-lying pair-addition vibration which we discussed in section IV and in Ref.[45]. In ${ }^{142-150} \mathrm{Sn}$, the pairing collective mode having a large transfer strength is only the pair rotation. (There exists low-lying monopole modes having very small strength in ${ }^{142-150} \mathrm{Sn}$ (cf Fig. 2), but we do not discuss these in this paper.)

From the systematic analysis, we find the following series and relations connecting the above mentioned various pair-transfer modes that appear in different regions of the $\mathrm{Sn}$ isotopes.

i) The giant pair vibration GPV2 in ${ }^{120-130} \mathrm{Sn}$, the second excited pair vibration PV2 in ${ }^{132} \mathrm{Sn}$, and the low-lying pair vibration in ${ }^{134-140} \mathrm{Sn}$. 
ii) The giant pair vibration GPV1 in ${ }^{120-130} \mathrm{Sn}$, the lowest pair vibration PV1 in ${ }^{132} \mathrm{Sn}$, and the pair rotation in ${ }^{134-140} \mathrm{Sn}$.

iii) The pair rotation in ${ }^{142-150} \mathrm{Sn}$ and the above two series i) and ii).

\section{A. Series i)}

We first discuss the series i). The forward amplitudes $X_{i i^{\prime}}^{\nu}$ of the GPV2 in ${ }^{120-130} \mathrm{Sn}$, the second excited pair vibration PV2 in ${ }^{132} \mathrm{Sn}$, and the low-lying pair vibration in ${ }^{134-140} \mathrm{Sn}$ are listed in Table III for the largest ten two-quasiparticle components.

It is seen that the $X$-amplitudes of the GPV2 are very similar for all the isotopes in the interval $A=120-130$. The largest is the two-quasiparticle configuration $\left[3 p_{3 / 2}\right]^{2}$ and the other largest components are $\left[2 f_{7 / 2}\right]^{2},\left[3 p_{1 / 2}\right]^{2},\left[1 h_{9 / 2}\right]^{2}\left[2 f_{5 / 2}\right]^{2}$ and $\left[1 i_{13 / 2}\right]^{2}$. The $X$ amplitudes of them all vary only gradually with changing the neutron number. A slight increase of collectivity is seen; for instance, the $X$-amplitude of $\left[1 i_{13 / 2}\right]^{2}$, the fifth largest in most cases, increases from 0.120 in ${ }^{120} \mathrm{Sn}$ to 0.193 in ${ }^{130} \mathrm{Sn}$. This is consistent with the increase of the pair-addition strength $B(\operatorname{Pad} 0)$ (cf . Fig. 2 and Table III).

We now compare the $X$-amplitudes of the GPV2 in ${ }^{128,130} \mathrm{Sn}$ with those of the second pair vibration PV2 in ${ }^{132} \mathrm{Sn}$ and the low-lying pair vibration in ${ }^{134} \mathrm{Sn}$. A similarity is obvious for the $X$-amplitudes of the largest components $\left[3 p_{3 / 2}\right]^{2},\left[2 f_{7 / 2}\right]^{2},\left[3 p_{1 / 2}\right]^{2},\left[1 h_{9 / 2}\right]^{2},\left[2 f_{5 / 2}\right]^{2}$ and $\left[1 i_{13 / 2}\right]^{2}$; the $X$-amplitudes are smoothly connected among the GPV2 in ${ }^{120-130} \mathrm{Sn}, \mathrm{PV} 2$ in ${ }^{132} \mathrm{Sn}$ and the low-lying pair vibration in ${ }^{134} \mathrm{Sn}$. Further increasing the neutron number to $A=136,138$, we find a continuation of the similarity for the low-lying pair-vibration in ${ }^{136,138} \mathrm{Sn}$. In $A=140$, we can still trace the same similarity to some extent, but at the same time the variation from $A=138$ to $A=140$ becomes slightly large.

Figure 9(a) shows the pair-addition transition densities for the series. We can confirm that the transition densities of the GPV2 modes in ${ }^{120-130} \mathrm{Sn}$ are smoothly connected to that for the pair-addition vibration in ${ }^{132-138} \mathrm{Sn}$.

\section{B. Series ii)}

The second series is identified among the GPV1 modes in ${ }^{120-130} \mathrm{Sn}$, the lowest pair vibration PV1 in ${ }^{132} \mathrm{Sn}$, and the pair rotations in the isotopes ${ }^{134-140} \mathrm{Sn}$. The latter two 


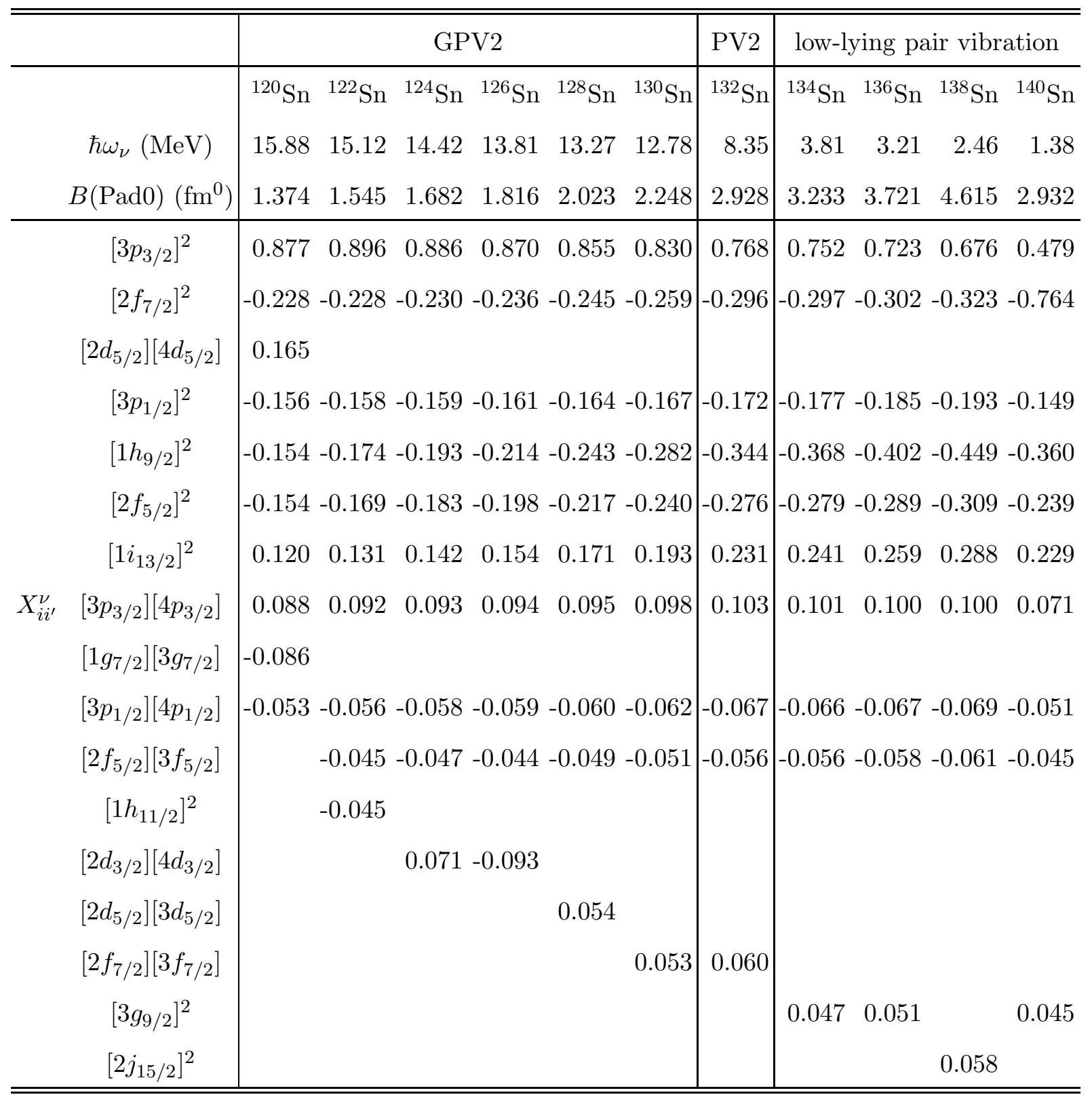

TABLE III: The forward amplitudes $X_{i i^{\prime}}^{\nu}$ of the giant pair vibration GPV2 in ${ }^{120-130} \mathrm{Sn}$, of the second excited pair vibration PV2 in ${ }^{132} \mathrm{Sn}$, and of the low-lying pair vibration in ${ }^{134-140} \mathrm{Sn}$. The two-quasiparticle components having ten largest values of $\left|X_{i i^{\prime}}^{\nu}\right|$ are listed for each mode. The excitation energy $\hbar \omega_{\nu}$ and the strength $B(\operatorname{Pad} 0 ; g s \rightarrow \nu)$ are also listed.

populates the ground states in neighboring $N+2$ isotopes.

We show in Table IV the $X$-amplitudes of two-quasiparticle components for the giant pair vibration GPV1 modes in ${ }^{120-130} \mathrm{Sn}$ and for the lowest pair-addition vibration PV1 in ${ }^{132} \mathrm{Sn}$. The main components include $\left[2 f_{7 / 2}\right]^{2},\left[1 h_{9 / 2}\right]^{2},\left[1 i_{13 / 2}\right]^{2},\left[2 f_{5 / 2}\right]^{2}$ and $\left[3 p_{3 / 2}\right]^{2}$ (they are 

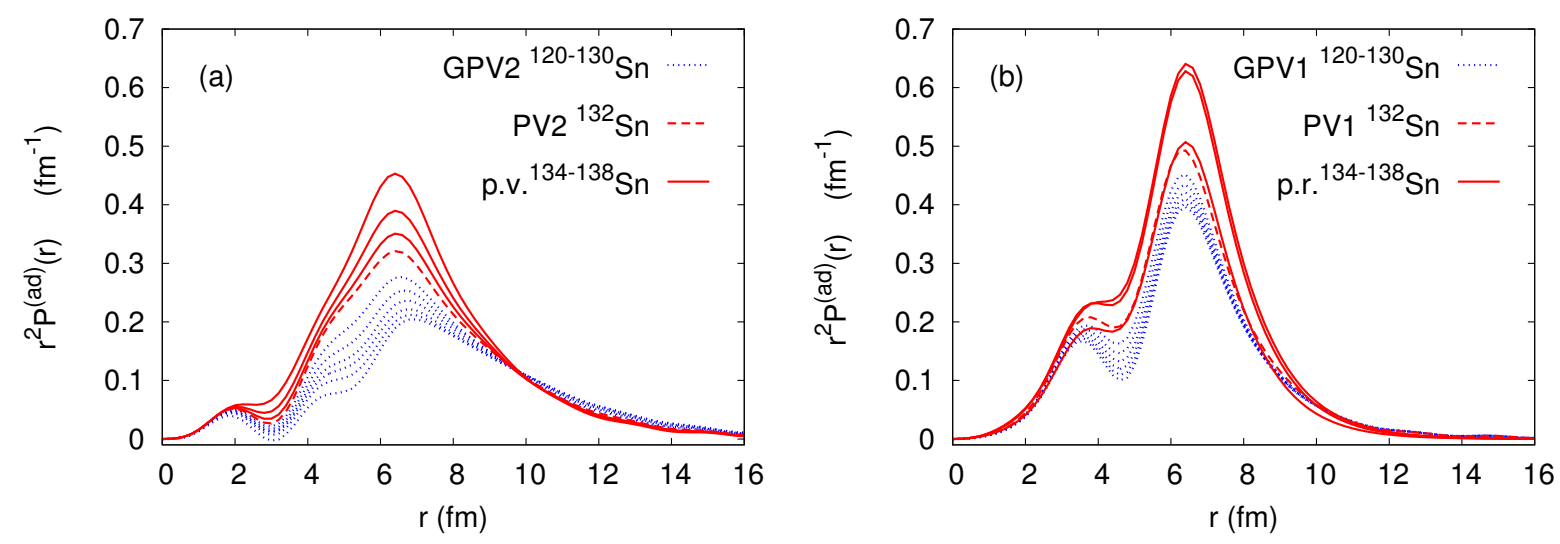

FIG. 9: (Color online) (a) The transition densities $r^{2} P_{\nu L}^{(\mathrm{ad})}(r)$ for the giant pair vibration GPV2 in $A=120-130$ (dotted), for the second pair vibration PV2 in $A=132$ (dashed) and for the low-lying pair vibration in $A=134-138$ (solid). (b) The transition densities for the giant pair vibration GPV1 in $A=120-130$ (dotted), for the first pair vibration PV1 in $A=132$ (dashed) and the transition densities of the pair rotation $r^{2} P_{\mathrm{gs}}^{(\mathrm{ad})}(r)$ in $A=134-138$ (solid).

all shared with the GPV2 mode ), and the largest is $\left[2 f_{7 / 2}\right]^{2}$ (this is different from GPV2). From the $X$-amplitudes of these components, we find that the GPV1 modes in ${ }^{120-130} \mathrm{Sn}$ have common microscopic structure with only small variation with $N$. It is seen also that the lowest pair vibration mode PV1 in ${ }^{132} \mathrm{Sn}$ has essentially the same microscopic structure with that of the GPV1 modes in ${ }^{120-130} \mathrm{Sn}$. This mode can be regarded as a smooth continuation of GPV1.

For the pair rotation modes in ${ }^{134-140} \mathrm{Sn}$, the $X$ - and $Y$-amplitudes are not evaluated. In place of them, we calculate decomposed transition densities $P_{\mathrm{gs}, i i}^{(\mathrm{ad})}(r)$ (Eq.(34)) for each of the main configurations $\left[2 f_{7 / 2}\right]^{2},\left[1 h_{9 / 2}\right]^{2},\left[1 i_{132}\right]^{2},\left[2 f_{5 / 2}\right]^{2}$ and $\left[3 p_{3 / 2}\right]^{2}$ of GPV1 in ${ }^{120-130} \mathrm{Sn}$. They are shown in Fig. 10(a). Comparing with the decomposed transition densities of GPV1, plotted in Fig. 6(b) for ${ }^{120} \mathrm{Sn}$, we find a close similarity between the two figures. We find also that the pair rotations in ${ }^{134-140} \mathrm{Sn}$ are all similar (the decomposed transition densities for ${ }^{136-140} \mathrm{Sn}$ are not shown here). It is seen in Fig. 9(b) that the transition densities of the GPV1 modes in ${ }^{120-130} \mathrm{Sn}$ smoothly match that of the PV1 in ${ }^{132} \mathrm{Sn}$, and those of the pair rotations in ${ }^{134-138} \mathrm{Sn}$. 


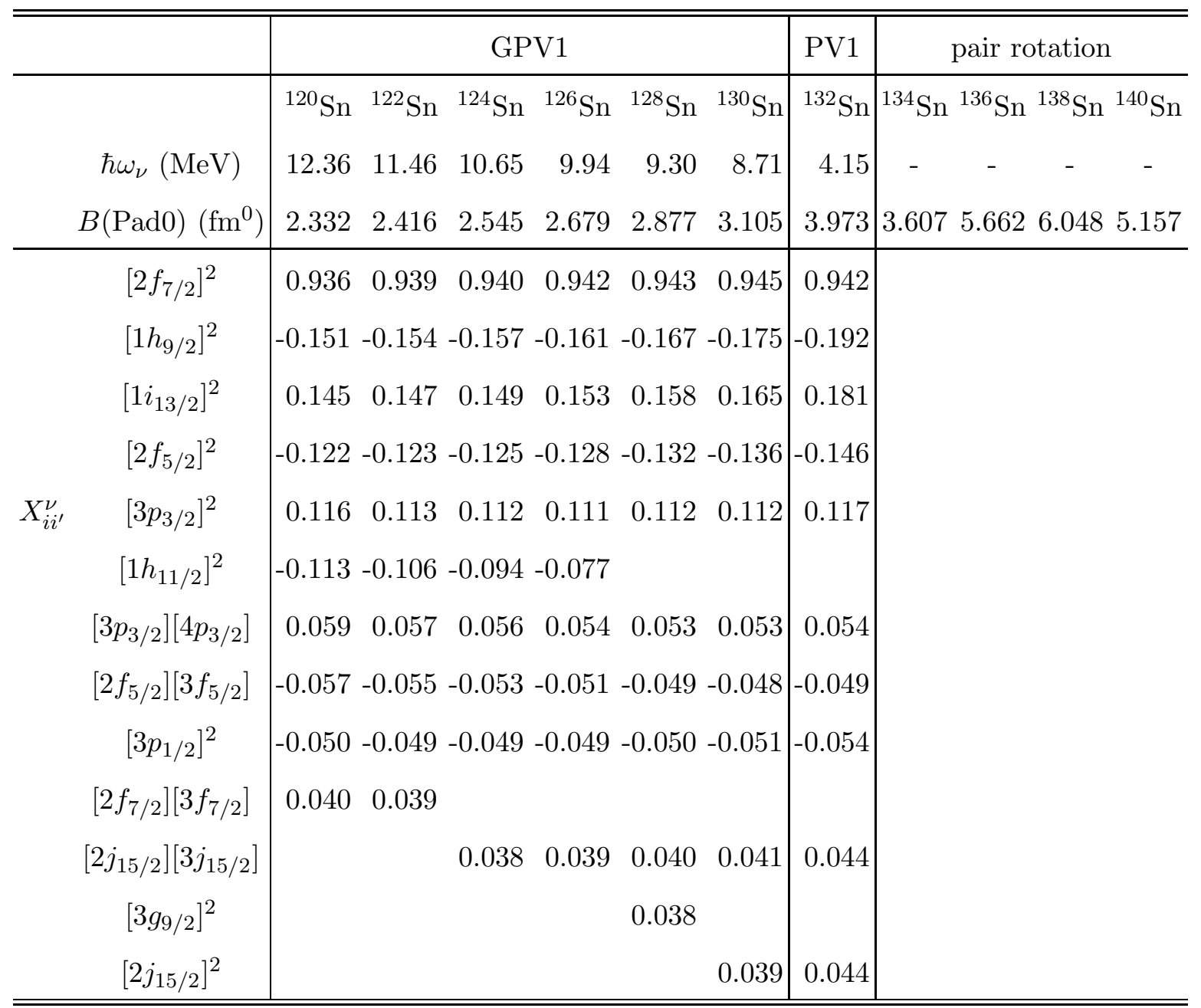

TABLE IV: The forward amplitudes $X_{i i^{\prime}}^{\nu}$ of the giant pair vibration GPV1 in ${ }^{120-130} \mathrm{Sn}$ and of the first pair vibration PV1 in ${ }^{132} \mathrm{Sn}$. The two-quasiparticle components having ten largest values of $\left|X_{i i^{\prime}}^{\nu}\right|$ are listed for each mode. The excitation energy $\hbar \omega_{\nu}$ and the strength $B(\operatorname{Pad} 0 ;$ gs $\rightarrow \nu)$ are also listed. For the pair rotation in ${ }^{134-140} \mathrm{Sn}$, we only list the strength $B(\operatorname{Pad} 0 ; \mathrm{gs} \rightarrow \mathrm{gs})$ since the $X$ - and $Y$-amplitudes are not evaluated.

\section{Relation iii)}

Finally we discuss the relation iii). As a representative of the pair rotational modes in ${ }^{142-150} \mathrm{Sn}$, we take ${ }^{142} \mathrm{Sn}$ and show in Fig. 10(b) microscopic decomposition of the pairaddition transition density $P_{\mathrm{gs}, i i}^{(\mathrm{ad})}(r)$ for the quasiparticle configurations $\left[3 p_{3 / 2}\right]^{2},\left[1 h_{9 / 2}\right]^{2}$, $\left[2 f_{7 / 2}\right]^{2},\left[2 f_{5 / 2}\right]^{2}$ and $\left[1 i_{13 / 2}\right]^{2}$. We now compare this with the decomposed transition densities of the low-lying pair vibration and the pair rotation modes in ${ }^{134} \mathrm{Sn}$ (Fig. 4(a) and Fig. 

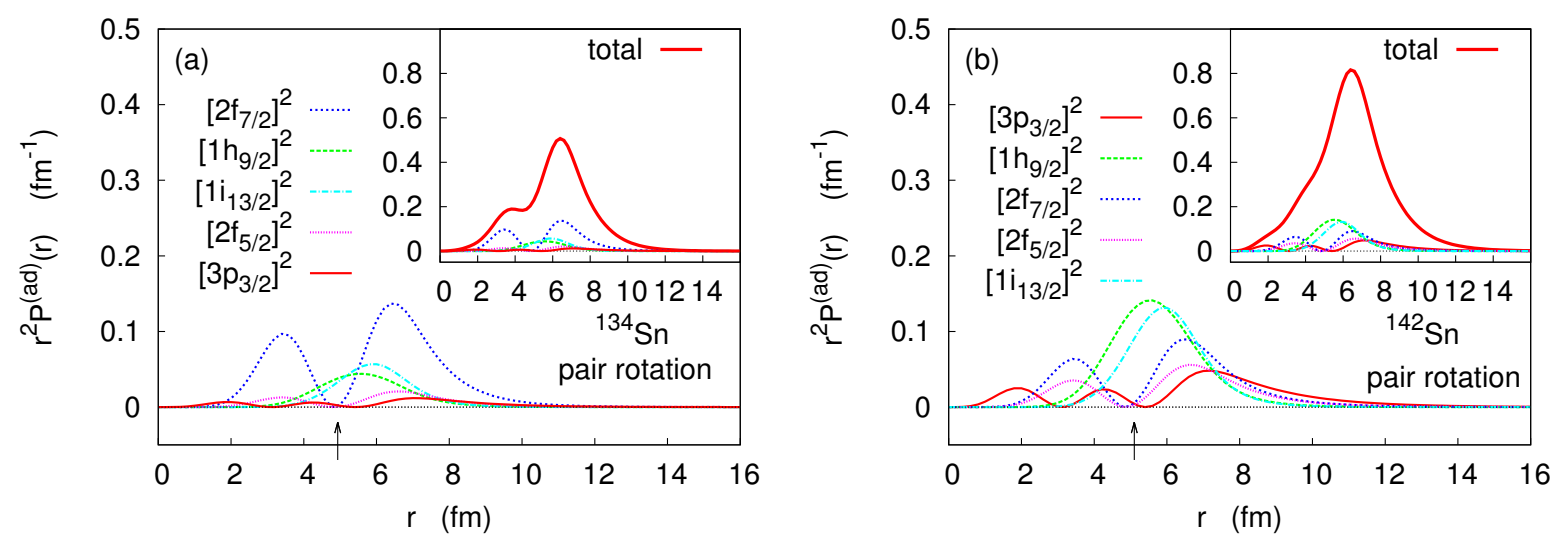

FIG. 10: (Color online) (a) The decomposed transition densities $P_{\mathrm{gs}, i i^{\prime}}^{(\mathrm{ad})}(r)$ of the components $\left[2 f_{7 / 2}\right]^{2},\left[1 h_{9 / 2}\right]^{2},\left[1 i_{13 / 2}\right]^{2},\left[2 f_{5 / 2}\right]^{2}$, and $\left[3 p_{3 / 2}\right]^{2}$ for the pair rotation in ${ }^{134} \mathrm{Sn}$. These five components correspond to those plotted in Fig. 6(b). The inset also shows the total transition density $P_{\mathrm{gs}}^{(\mathrm{ad})}(r)$. The arrow indicates the neutron rms radius $R_{N, r m s}$. (b) The same as (a) but for the pair rotation in ${ }^{142} \mathrm{Sn}$. The components plotted are $\left[3 p_{3 / 2}\right]^{2},\left[1 h_{9 / 2}\right]^{2},\left[2 f_{7 / 2}\right]^{2},\left[2 f_{5 / 2}\right]^{2}$ and $\left[1 i_{13 / 2}\right]^{2}$.

10(a)). It is not difficult to find some similarity between the transition density of the pair rotation in ${ }^{142} \mathrm{Sn}$ (Fig. 10(b)) and that of the low-lying pair vibration in ${ }^{134} \mathrm{Sn}$ (Fig. 4(a)). To be more specific, the components which have the largest amplitudes at $r \sim 6 \mathrm{fm}$ are the quasiparticle states $\left[1 h_{9 / 2}\right]$ and $\left[1 i_{13 / 2}\right]$ in both cases. The largest contribution in the external region $r \gtrsim 8 \mathrm{fm}$ is that of the quasiparticle state $\left[3 p_{3 / 2}\right]$. However, a difference is seen in the contribution of $\left[2 f_{7 / 2}\right]$, which has positive amplitude for the pair rotation in ${ }^{142} \mathrm{Sn}$ (Fig. 10(b)), but the phase is opposite for the low-lying pair vibration in ${ }^{134} \mathrm{Sn}$ (Fig. 4(a)). We then compare the pair rotation in ${ }^{134} \mathrm{Sn}$ (Fig. 10(a)) and that in ${ }^{142} \mathrm{Sn}$ (Fig. 10(b)), and find that the $\left[2 f_{7 / 2}\right]$ amplitude is positive and large in both cases. The above observations suggest that, if we superpose the low-lying pair vibration and the pair rotation in ${ }^{134} \mathrm{Sn}$, a resultant transition density may resemble to that of the pair rotation in ${ }^{142} \mathrm{Sn}$. The analysis of other isotopes ${ }^{136-140} \mathrm{Sn}$, not shown here, also suggests the same feature. We thus deduce that the two pairing collectivities in ${ }^{134-140} \mathrm{Sn}$, i.e. the series i) and ii), merge into a single mode, which appears as the pair rotation in ${ }^{142-150} \mathrm{Sn}$. 


\section{CONCLUSIONS}

We have investigated the monopole pair-additional transfer modes using the SkyrmeHartree-Fock-Bogoliubov theory and the linear response formalism of QRPA using the Skyrme parameter set SLy4 and the DDDI pairing interaction. We particularly analyzed the microscopic structure of the pairing collective modes in detail by evaluating the forward and backward amplitudes $X_{i i^{\prime}}^{\nu}$ and $Y_{i i^{\prime}}^{\nu}$ of the QRPA phonon operator, and by decomposing the transition densities associated with the pair-addition transfer operator. We apply this analysis for the low-lying pair vibration, the high-lying giant pair vibration and the pair rotation in even-even $A=120-150$ Sn isotopes.

We have first investigated the low-lying pair-addition vibration emerging in neutronrich ${ }^{134-140} \mathrm{Sn}$ in order to reveal its very large pair-addition strength[45]. The largest twoquasiparticle components of this mode are those involving the neutron quasiparticle states located just above the $N=82$ shell gap, i.e. $2 f_{7 / 2}, 3 p_{3 / 2}, 3 p_{1 / 2}, 2 f_{5 / 2}, 1 h_{9 / 2}$ etc, but it turned out that contributions from these largest components can account for only a part of the amplitude and the long tail of the transition density. We found significant contributions from the quasiparticle states which have larger orbital angular momenta $l>5$, reaching to $l \gtrsim 10$, all of which are unbound continuum states. These two-quasiparticle configurations of high- $l$ states accumulate coherently to build up the large and extended tail of the transition density. This suggests that a neutron pair transfered in the low-lying pair-addition vibration of ${ }^{134-140}$ Sn exhibits a strong spatial correlation, the di-neutron correlation, especially outside the nuclear surface.

We have analyzed also isotopes ${ }^{110-130} \mathrm{Sn}$ in order to explore the long-tail pairing vibration in isotopes closer to the stability line. The present calculation predicts presence of the giant pair vibrations, with two peaks GPV1 and GPV2, in $110 \lesssim A \leq 130$ with excitation energy $\hbar \omega_{\nu} \approx 8-20 \mathrm{MeV}$. The pair-addition strengths of GPV1 and GPV2 are comparable to (but slightly smaller than) that of the low-lying pair vibration in ${ }^{134-140} \mathrm{Sn}$. The detailed analysis of the phonon amplitudes and the transition density revealed that these modes have similar microscopic structures as that of the low-lying pair vibration in ${ }^{134-140} \mathrm{Sn}$, and the di-neutron character and the long tail are also seen. The di-neutron character is also seen in the pair-addition vibrations in ${ }^{132} \mathrm{Sn}$ and the pair rotation in ${ }^{134-150} \mathrm{Sn}$.

From the systematic analysis performed for all the isotopes from the stable ones to very 
neutron-rich ${ }^{150} \mathrm{Sn}$, we found the above pairing collective modes are all related. The giant pair vibration GPV1 in ${ }^{110-130} \mathrm{Sn}$ is smoothly connected to the lowest pair-addition vibration in ${ }^{132} \mathrm{Sn}$ (the lowest one populating the ground state of ${ }^{134} \mathrm{Sn}$ ), which is then connected to the pair rotation in ${ }^{134-140} \mathrm{Sn}$. A parallel series is the GPV2 in ${ }^{120-130} \mathrm{Sn}$, the second lowest pair-addition vibration in ${ }^{132} \mathrm{Sn}$, and the low-lying pair vibration in ${ }^{134-140} \mathrm{Sn}$, which are also connected smoothly with changing $N$. At ${ }^{142} \mathrm{Sn}$, these two series merge into a single mode, the pair rotation, which then continues further for $A>142$. The tail enhancement in the transition density and the collectivity in the pair-addition strength of these modes increase constantly with $N$, especially at $A>132$ where the neutron Fermi energy becomes very shallow.

\section{ACKNOWLEDGMENTS}

The authors thank K. Yoshida for useful advises and discussions. The authors also thank K. Matsuyanagi, K. Yabana, T. Nakatsukasa and H. Liang for valuable discussions. The numerical calculations were performed partly on the HITACHI SR1600 supercomputer systems at Yukawa Institute for Theoretical Physics, Kyoto University. This work is supported by Grants-in-Aid for Scientific Research (No. 21340073, No. 23540294, No.24105008, and No.25287065) from the Japan Society for the Promotion of Science.

[1] I. Tanihata et al., Phys. Rev. Lett.55, 2676 (1985).

[2] I. Tanihata, Prog. Part. Nucl. Phys. 35, 505 (1995).

[3] G. F. Bertsch and H. Esbensen, Ann. Phys. (NY) 209, 327,(1991).

[4] J. Dobaczewski,W. Nazarewicz and P.-G. Reinhard, Nucl. Phys. A 693, 361 (2001).

[5] M. Matsuo and T. Nakatsukasa, J. Phys. G: Nucl. Part. Phys. 37, 064017 (2010).

[6] U. Lombardo and H.-J. Schulze, in Physics of Neutron Star Interiors, edited by D. Blaschke, N. K. Glendenning, and A. Sedrakian (Springer, New York, 2001), Lecture Notes in Physics, Vol. 578 , p. 30 .

[7] D. J. Dean and M. Hjorth-Jensen, Rev. Mod. Phys. 75, 607 (2003).

[8] M. Matsuo, Phys. Rev. C 73, 044309 (2006). 
[9] J. Margueron, H. Sagawa and K. Hagino, Phys. Rev. C 77, 054309 (2008).

[10] A. Gezerlis and J. Carlson, Phys. Rev. C 81, 025803 (2010).

[11] P. G. Hansen and B. Jonson, Europhys. Lett. 4, 409 (1987).

[12] H. Esbensen and G. F. Bertsch, Nucl. Phys. A 542, 310 (1992).

[13] K. Ikeda, Nucl. Phys. A 538, 355c (1992).

[14] M. V. Zhukov, B. V. Danilin, D. V. Fedorov, J. M. Bang, I. J. Thompson and J. S. Vaagen, Phys. Rep. 231, 151 (1993).

[15] K. Hagino and H. Sagawa, Phys. Rev. C 72, 044321 (2005).

[16] S. Aoyama, T. Myo, K. Katō, and K. Ikeda, Prog. Theor. Phys. 116, 1 (2006).

[17] Y. Kanada-En'yo Phys. Rev. C 76, 044323 (2007).

[18] T. Myo, Y. Kikuchi, K. Katō, and K. Ikeda, Prog. Theor. Phys. 119, 561 (2008).

[19] M. Matsuo, K. Mizuyama and Y. Serizawa, Phys. Rev. C 71, 064326 (2005).

[20] M. Matsuo, H. Shimoyama and Y. Ootaki, Phys. Scr. T 150, 014024 (2012).

[21] M. Matsuo, in Fifty Years Nuclear BCS Pairing in Finite Systems, edited by R. A. Broglia and V. Zelevinsky (World Scientific, Singapore 2013), p. 61.

[22] N. Pillet, N. Sandulescu and P. Schuck, Phys. Rev. C 76, 024310 (2007).

[23] N. Pillet, N. Sandulescu, P. Schuck and J.-F. Berger, Phys. Rev. C 81, 034307 (2010).

[24] R. H. Ibarra, N. Austern, M. Vallieres, and D. H. Feng, Nucl. Phys. A 288, 397 (1977).

[25] F. Catara, A. Insolia, E. Maglione, and A. Vitturi, Phys. Rev. C 29, 1091 (1984).

[26] L. Ferreira, R. Liotta, C. H. Dasso, R. A. Broglia, and A. Winther, Nucl. Phys. A 426, 276 (1984).

[27] T. Nakamura, et al. Phys. Rev. Lett. 96, 252502 (2006).

[28] Y. Serizawa and M. Matsuo, Prog. Theor. Phys. 121, 97 (2009).

[29] F. Kobayashi and Y. Kanada-En'yo, Phys. Rev. C 86, 064303 (2012).

[30] D. R. Bes and R. A. Broglia, Nucl. Phys. 80, 289 (1966).

[31] R. A. Broglia, O. Hansen and C. Riedel, in Advances in Nuclear Physics, edited by M. Baranger and E. Vogt (Plenum, New York, 1973), Vol. 6, p. 287.

[32] D. M. Brink and R. A. Broglia, Nuclear Superfluidity: Pairing in Finite Systems (Cambridge University Press, Cambridge, 2005).

[33] S. Yoshida, Nucl. Phys. 33, 685 (1962).

[34] A. Bohr and B. R. Mottelson, Nuclear Structure, (Benjamin, New York, 1998), Vol. II. 
[35] N. Keeley et al., Phys. Lett. B 646, 222 (2007).

[36] I. Tanihata et al., Phys. Rev. Lett. 100, 192502 (2008).

[37] A. Chatterjee et al., Phys. Rev. Lett. 101, 032701 (2008).

[38] M. S. Golovkov et al., Phys. Lett. B 672, 22 (2009).

[39] K. Wimmer et al., Phys. Lett. B 105, 252501 (2010).

[40] A. Lemasson et al., Phys. Lett. B 697, 454 (2011).

[41] E. Khan, N. Sandulescu, N. V. Giai, and M. Grasso, Phys Rev. C 69, 014314 (2004).

[42] B. Avez, C. Simenel, and Ph. Chomaz, Phys. Rev. C 78, 044318 (2008).

[43] E. Khan, M. Grasso, and J. Margueron, Phys Rev. C 80, 044328 (2009).

[44] M. Matsuo and Y. Serizawa, Phys. Rev. C 82, 024318 (2010).

[45] H. Shimoyama and M. Matsuo, Phys. Rev. C 84, 044317 (2011).

[46] G. Potel, F. Barranco, F. Marini, A. Idini, E. Vigezzi and R. A. Broglia, Phys. Rev. Lett. 107, 092501 (2011); Phys. Rev. Lett. 108, 069904(E) (2012).

[47] M. Grasso, D. Lacroix, A. Vitturi, Phys. Rev. C 85, 034317 (2012).

[48] G. Potel, A. Idini, F. Barranco, E. Vigezzi and R. A. Broglia, Phys. Rev. C 87, 054321 (2013).

[49] M. Matsuo, Nucl. Phys. A 696, 371 (2001): Prog. Theor. Phys. Suppl. 146, 110 (2002).

[50] P. Ring and P. Schuck, The Nuclear Many-Body Problem, (Springer-Verlag, Berlin, 1980).

[51] E. Chabanat, P. Bonche, P. Haensel, J. Meyer and R. Schaeffer, Nucl. Phys. A 635, 231 (1998); 643, 441 (1998).

[52] R. R. Chasman, Phys. Rev. C 14, 1935 (1976).

[53] E. Garrido, P. Sarriguren, E. Moya de Guerra, U. Lombardo, P. Schuck and H. J. Schulze, Phys. Rev. C 63, 037304 (2001).

[54] W. Satula, J. Dobaczewski, and W. Nazarewicz, Phys. Rev. Lett. 81, 3599 (1998).

[55] W. von Oertzen and A. Vitturi, Rep. Prog. Phys. 64, 1247 (2001).

[56] R. A. Broglia and D. R. Bes, Phys. Lett. B 69, 129 (1977).

[57] P. Lotti, F. Cazzola, P. F. Bortignon, R. A. Broglia and A. Vitturi, Phys. Rev. C 40, 1791 (1989).

[58] M. W. Herzog, R. J. Liotta and L. J. Sibanda, Phys. Rev. C 31, 259 (1985). 